Louisiana State University

LSU Digital Commons

Faculty Publications

Department of Biological Sciences

$12-24-2019$

\title{
Characterization of an Extensive Interface on Vitronectin for Binding to Plasminogen Activator Inhibitor-1: Adoption of Structure in an Intrinsically Disordered Region
}

\author{
Letitia O. Puster \\ The University of Tennessee, Knoxville \\ Christopher B. Stanley \\ Oak Ridge National Laboratory \\ Vladimir N. Uversky \\ Morsani College of Medicine \\ Joseph E. Curtis \\ National Institute of Standards and Technology \\ Susan Krueger \\ National Institute of Standards and Technology
}

See next page for additional authors

Follow this and additional works at: https://digitalcommons.Isu.edu/biosci_pubs

\section{Recommended Citation}

Puster, L., Stanley, C., Uversky, V., Curtis, J., Krueger, S., Chu, Y., \& Peterson, C. (2019). Characterization of an Extensive Interface on Vitronectin for Binding to Plasminogen Activator Inhibitor-1: Adoption of Structure in an Intrinsically Disordered Region. Biochemistry, 58 (51), 5117-5134. https://doi.org/10.1021/ acs.biochem.9b00605

This Article is brought to you for free and open access by the Department of Biological Sciences at LSU Digital Commons. It has been accepted for inclusion in Faculty Publications by an authorized administrator of LSU Digital Commons. For more information, please contact ir@lsu.edu. 


\section{Authors}

Letitia O. Puster, Christopher B. Stanley, Vladimir N. Uversky, Joseph E. Curtis, Susan Krueger, Yuzhuo Chu, and Cynthia B. Peterson 


\title{
Characterization of an Extensive Interface on Vitronectin for Binding to Plasminogen Activator Inhibitor-1: Adoption of Structure in an Intrinsically Disordered Region
}

\author{
Letitia O. Puster, ${ }^{\dagger}$ Christopher B. Stanley, ${ }^{\ddagger}$ Vladimir N. Uversky, ${ }^{\S} \|_{\odot}$ Joseph E. Curtis, ${ }^{\perp}$ \\ Susan Krueger, ${ }^{\perp}$ Yuzhuo Chu, ${ }^{\#}$ and Cynthia B. Peterson ${ }^{*},{ }^{\infty}$
}

${ }^{\dagger}$ Department of Biochemistry and Cellular and Molecular Biology, University of Tennessee, Knoxville, Tennessee 37996, United States

${ }^{\ddagger}$ Computational Sciences and Engineering Division, Oak Ridge National Laboratory, Oak Ridge, Tennessee 37831, United States

${ }^{\S}$ Department of Molecular Medicine and USF Health Byrd Alzheimer's Research Institute, Morsani College of Medicine, University of South Florida, Tampa, Florida 33612, United States

"Laboratory of New Methods in Biology, Institute for Biological Instrumentation, Russian Academy of Sciences, Pushchino, Moscow region 142290, Russia

${ }^{\perp}$ National Institute of Standards and Technology Center for Neutron Research, Gaithersburg, Maryland 20899, United States

\#Department of Biological Sciences, Louisiana State University, Baton Rouge, Louisiana 70803, United States

Supporting Information

ABSTRACT: Small-angle neutron scattering (SANS) measurements were pursued to study human vitronectin, a protein found in tissues and the circulation that regulates cell adhesion/ migration and proteolytic cascades that govern hemostasis and pericellular proteolysis. Many of these functions occur via interactions with its binding partner, plasminogen activator inhibitor-1 (PAI-1), the chief inhibitor of proteases that lyse and activate plasminogen. We focused on a region of vitronectin that remains uncharacterized from previous X-ray scattering, nuclear magnetic resonance, and computational modeling approaches and which we propose is involved in binding to PAI-1. This region, which bridges the N-terminal somatomedin B (SMB) domain with a large central $\beta$-propeller domain of vitronectin, appears unstructured and has characteristics of an intrinsically disordered domain (IDD). The effect of osmolytes was evaluated using circular dichroism and SANS to explore the potential of the IDD to undergo a disorder-to-order transition. The results suggest that the IDD favors a more ordered structure under osmotic pressure; SANS shows a smaller radius of gyration $\left(R_{\mathrm{g}}\right)$ and a more compact fold of the IDD upon addition of osmolytes. To test whether PAI-1 binding is also coupled to folding within the IDD structure, a set of SANS experiments with contrast variation were performed on the complex of PAI-1 with a vitronectin fragment corresponding to the N-terminal 130 amino acids (denoted the SMB-IDD because it contains the SMB domain and IDD in linear sequence). Analysis of the SANS data using the Ensemble Optimization Method confirms that the SMB-IDD adopts a more compact configuration when bound to PAI-1. Calculated structures for the PAI-1:SMB-IDD complex suggest that the IDD provides an interaction surface outside of the primary PAI-1-binding site located within the SMB domain; this binding is proposed to lead to the assembly of higher-order structures of vitronectin and PAI-1 commonly found in tissues.

$\mathrm{V}$ itronectin (VN) was originally identified as a component in serum that promoted adhesion of mammalian cells to culture vessels. ${ }^{3,4}$ Antibody probes were used to show that VN is also localized to cell surfaces and is abundant in the extracellular matrix (ECM). ${ }^{5,6} \mathrm{VN}$ has multiple binding partners, including plasminogen, ${ }^{7}$ fibrinogen, ${ }^{8}$ several integrins, ${ }^{9-11}$ urokinase plasminogen activator receptor, ${ }^{13}$ heparin, ${ }^{14}$ and plasminogen activator inhibitor-1 (PAI-1). ${ }^{15}$ These partners affect the structure and function of $\mathrm{VN}$; in turn, binding to $\mathrm{VN}$ can alter the structure, activity, and localization of several of its binding partners. ${ }^{16-19}$

While there has been much interest in how vitronectin function relates to the folding of this multidomain protein, the full-length structure has not been determined. During biosynthesis, removal of a 19-residue signal peptide yields the mature vitronectin chain of 459 amino acids (72 kDa); further processing of mature vitronectin by proteolytic cleavage at position 379 can produce a disulfide cross-linked form comprising 62 and $10 \mathrm{kDa}$ subunits. A genetic polymorphism encoding Thr or Met as residue 381 controls the propensity of vitronectin to be cleaved to the two-chain form; ${ }^{20}$ however, the single- and two-chain variants appear to be functionally

Received: July 14, 2019

Revised: November 29, 2019

Published: December 3, 2019 
a

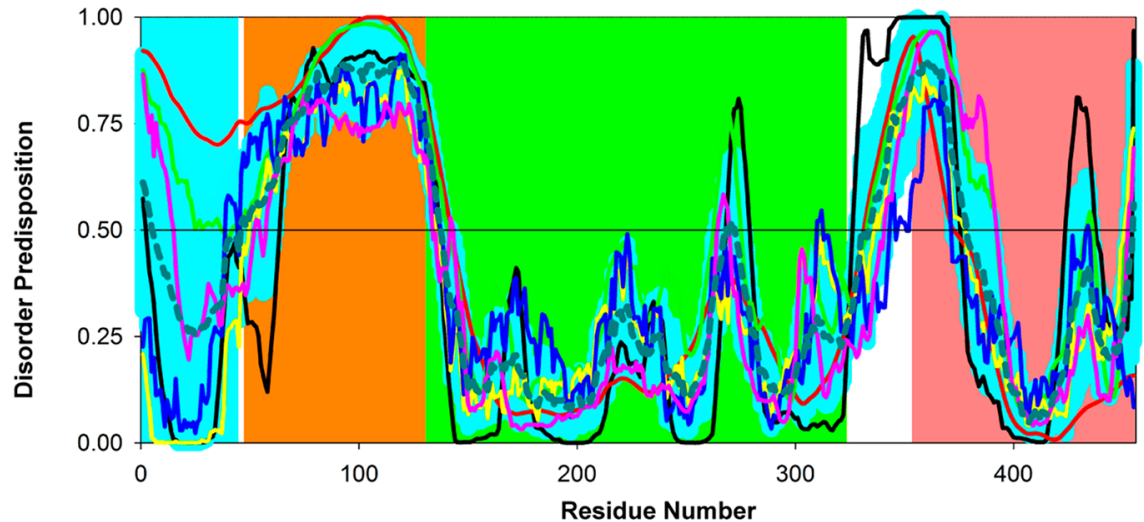

SMB Domain IDD

Central Domain

C-terminal Domain

\begin{tabular}{|l|l|l|l|}
\hline $1-44$ & $48-130$ & $131-323$ & $354-456$ \\
\hline
\end{tabular}

b

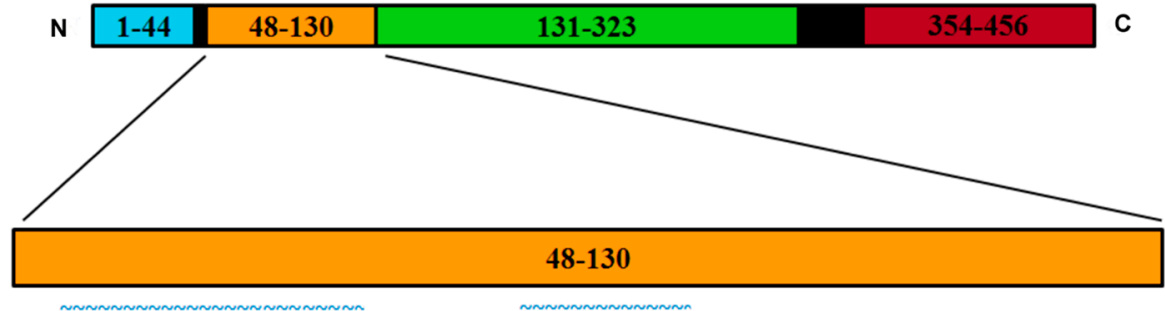

VFT MPEDEYTVYD DGEEKNNATV HEQVGGPSLT SDLQAQSKGN PEQTPVLKPE EEAPAPEVGA SKPEGIDSRP ETLHPGRPQP

Figure 1. Analysis of the intrinsic disorder predisposition of human vitronectin and the potential of the IDD to adopt structure. The domain organization of vitronectin is shown in the linear structure between panels and a and $\mathrm{b}{ }^{1}{ }^{1}$ The $\mathrm{N}$-terminal somatomedin $\mathrm{B}$ domain is colored blue, the central domain characterized by a $\beta$-propeller structure green, and the $\mathrm{C}$-terminal domain corresponding to a half-propeller structure red. The proposed IDD corresponding to the linker region (residues 48-130) is highlighted in amber. (a) Evaluating the intrinsic disorder propensity of human VN (UniProt ID P04004). Disorder propensity was evaluated by PONDR VLXT (black curve), PONDR VL3 (red curve), PONDR VSL2 (green curve), PONDR FIT (pink curve), IUPred_short (yellow curve), and IUPred_long (blue curve). The DisProt database (http://original. disprot.org $/)^{2}$ was the source used to calculate the per-residue disorder predictors of the PONDR family. The results for the per-residue disorder propensity calculated by these tools are given as real numbers between 1 (ideal prediction of disorder) and 0 (ideal prediction of order). A threshold of $\geq 0.5$ was used to identify disordered residues/regions in vitronectin, whereas those with disorder scores ranging from 0.2 to 0.5 are classified as flexible. Mean disorder predisposition was calculated by averaging all predictor-specific per-residue disorder profiles (bold, dashed, dark cyan curve). The light blue shadow around the mean disorder curve shows the distribution of standard deviations. Shaded areas represent positions of various VN domains. (b) Evaluation of the IDD sequence identified regions that may adopt helical structure. The amino acid sequence is shown below the expanded IDD from the main schematic representation of the multidomain structure of VN. The two segments of the IDD identified by the MoRF prediction software ${ }^{12}$ that may assume an $\alpha$-helical structure upon binding are colored blue.

indistinguishable. $^{21} \mathrm{X}$-ray scattering and computational predictions for the full-length protein, along with experimental structures determined for the $\mathrm{N}$-terminal somatomedin $\mathrm{B}$ (SMB) domain, have led to a proposed domain organization and three-dimensional models for vitronectin. ${ }^{1,22-26}$ This domain structure is illustrated in Figure 1. The SMB domain within vitronectin corresponds to its first 44 amino acids, with a partial $3_{10}$-helix and a single turn $\alpha$-helix as the only classical secondary structural elements; the remainder consists of unstructured sequences (loops and coils) connected by four disulfide bonds. The third and fourth domains of $\mathrm{VN}$, denoted the central domain (amino acids 131-342) and the C-terminal domain (amino acids 347-456), respectively, feature high $\beta$ structure content. Computational predictions indicate that the central domain adopts a $\beta$-propeller structure and the $\mathrm{C}$ terminal domain assumes a $\beta$-blade (half-propeller) fold. ${ }^{1,27}$

Between the first and third domains lies a second region, which is an $\sim 80$-amino acid stretch that contains no predicted secondary structure. This linker region is characterized by features typically associated with an intrinsically disordered domain. $^{28,29}$ These characteristics generally include the existence of low sequence complexity and bias in amino acid content; in particular, these regions typically house a large number of polar and charged amino acids (Gln, Glu, Lys, Pro, and Ser) but have few bulky hydrophobic residues (Ile, Leu, Met, Phe, Trp, Tyr, and Val). ${ }^{30,31}$ Investigations of intrinsically disordered regions within proteins have revealed noteworthy structure-function properties. ${ }^{32,33}$ Often, intrinsically disordered proteins or domains adopt secondary structures or threedimensional conformations that are induced upon binding to partner proteins/ligands. ${ }^{34}$ Barring the adoption of specific secondary structure, disordered regions can also interact with binding partners via "disordered" loops. The versatility of these disordered regions and their ability to adopt a variety of folds when interacting with different partners often lead to diversity in binding targets. ${ }^{35,36}$ Evaluating features of the linker region of $\mathrm{VN}$ as an intrinsically disordered region can provide new insights into the role of this domain in physiological functions.

When VN interacts with PAI-1, an irreversible structural change in $\mathrm{VN}$ occurs, leading to the accumulation of $\mathrm{VN}$ oligomers. ${ }^{37}$ Work over many years has shown that PAI-1- and VN-binding surfaces are extensive, and some have proposed that two distinct binding sites exist for these proteins. The primary binding sites on PAI-1 and VN are well-characterized. ${ }^{38,39}$ These involve interactions between the flexible joint region in PAI-1, containing helices $\mathrm{D}-\mathrm{F}$, and the $\mathrm{SMB}$ domain of $\mathrm{VN}^{38,40,41}$ Progress was made recently in our laboratory using mutagenesis and monoclonal antibodies to identify a 
more expansive binding interface that houses a separate binding site for VN on PAI-1. ${ }^{41}$ This site contains several basic residues and overlaps in part with the locus for the binding of heparin to PAI- $1 .^{41}$ However, the location of a corresponding second binding site for PAI-1 on VN has not been resolved. ${ }^{42}$ In early studies, two different regions were suggested to house such a second PAI-1-binding site, but neither has been substantiated in further work. One notion was that the site is located within the heparin-binding domain, which includes residues 348-370 within the C-terminal domain of $\mathrm{VN},{ }^{43,44}$ whereas a different binding site was proposed in the vicinity of amino acids $115-121$. $^{45}$

In this study, our goal was to characterize the secondary binding site within vitronectin. We hypothesize that the linker region of vitronectin is an intrinsically disordered domain (IDD) that houses the additional binding site for PAI-1 on VN. We also hypothesize that this IDD region undergoes a transition from a disordered to a more ordered structure when PAI-1 binds to VN. We note the number of negatively charged residues within the IDD (sequence given in Figure $1 \mathrm{~b}$ ) that could form ionic bonds with basic residues in the extensive binding site recently characterized in PAI- $1 .{ }^{41}$ Furthermore, the flexibility of the IDD is compatible with the hypothesis that the primary and secondary binding sites of $\mathrm{VN}$ are proximal to one another. This study employs sophisticated structural methods to determine the effect of PAI-1 binding on the structure of this linker region within $\mathrm{VN}$. In a recent complementary investigation, we used truncated forms of vitronectin (SMB-IDD fragments) to pursue kinetic analyses that support this assignment for the secondary binding site. ${ }^{46}$ Our discoveries advance the field by providing structural information about an understudied domain of $\mathrm{VN}$ and generating models to understand how PAI-1 interactions in this region are key within the multistep pathway of assembly of PAI-1:vitronectin complexes that associate into higher-order forms and display altered cell binding and adhesion.

\section{MATERIALS AND METHODS ${ }^{A}$}

Bacterial Expression and Purification of the SMB-IDD Fragment. Amino acids $1-130$ of VN (the SMB-IDD fragment) were engineered into the pET-32b plasmid, containing thioredoxin and six-histidine tags. The polyhistidine tag was included to aid in purification by immobilized metal affinity chromatography (IMAC). The thioredoxin tag was included to prevent accumulation of recombinant protein fragments in inclusion bodies and thus improve solubility. ${ }^{47}$ The pET-32b-SMB-IDD plasmid was transformed into Rosetta gami2(DE3) pLysS Escherichia coli cells, and SMB-IDD was expressed and purified using previously published procedures. ${ }^{48}$ After the cell pellet had been collected and lysed, the soluble cell lysate was chromatographed on an IMAC column charged with nickel, using a linear gradient from 20 to $1000 \mathrm{mM}$ imidazole for elution. Fractions that contained the vitronectin fragment fused with thioredoxin were collected and enzymatically digested with thrombin. After protease treatment, the protein digest was subjected to a second separation on the nickel-charged IMAC column, to which the fusion tags remained bound. The fractions that did not bind to the IMAC column were then loaded on an affinity column prepared with bound W175F PAI-1 to select the properly folded vitronectin fragment, which bound to the column, as described elsewhere. ${ }^{48}$ Sodium dodecyl sulfate-polyacrylamide gel electrophoresis (SDS-PAGE), Western blotting, and mass spectrom- etry (MALDI-TOF-MS) were utilized to determine size, identity, and purity during protein purification.

Expression and Purification of Deuterated PAI-1. Deuterated PAI-1 was prepared using two different approaches in this study. Glycerol stocks of E. coli containing PAI-1 cDNA in a pET-32b vector were first grown overnight in $10-15 \mathrm{~mL}$ of deuterated medium (TB with $50 \mu \mathrm{g} / \mathrm{mL}$ kanamycin and 34 $\mu \mathrm{g} / \mathrm{mL}$ chloramphenicol) to generate starter cultures. The following morning, these starter cultures provided the inoculum for $4 \mathrm{~L}$ of deuterated medium. The large cultures of deuterated protein were grown while being shaken at 220 $\operatorname{rpm}(1 \mathrm{rpm}=0.0167 \mathrm{~Hz})$ at $37^{\circ} \mathrm{C}$ until the $\mathrm{OD}_{600}$ reached $\sim 2$ and then cooled to $15{ }^{\circ} \mathrm{C}$ prior to induction of protein expression by addition of $1 \mathrm{mM}$ isopropyl $\beta$-D-1-thiogalactopyranoside (IPTG). Eighteen hours after induction, cultures were harvested by centrifugation to isolate cell pellets, which were frozen at $-80{ }^{\circ} \mathrm{C}$ until protein purification commenced.

Fermentation in minimal medium using the Biodeuteration lab at Oak Ridge National Laboratory was also used to more efficiently and cost effectively deuterate PAI-1. Starter cultures were grown, as described above, in a $70 \%$ deuterated minimal medium. An inoculum from the starter cultures was added to a $1 \mathrm{~L}$ culture, and cells were grown to an $\mathrm{OD}_{600}$ of $\sim 35$. The culture was cooled to $15{ }^{\circ} \mathrm{C}$, and IPTG was added to induce protein expression with overnight incubation as described above. On the basis of the starting level of $\mathrm{D}_{2} \mathrm{O}$ in the medium, the final level of deuteration of PAI-1 was expected to be $60-$ $65 \%$.

For these experiments, a form of PAI-1 with tryptophan 175 replaced with phenylalanine (W175F) was utilized because of its known slow rate of refolding from an active conformation to the inactive, latent form. ${ }^{49}$ Deuterated W175F PAI-1 was purified as previously described, ${ }^{48}$ using a three-step protocol that includes cation exchange chromatography using SPSepharose, IMAC using a nickel-charged resin, and finally size exclusion chromatography on a Sephacryl S-100 column. All buffers were prepared in $\mathrm{H}_{2} \mathrm{O}$, and the deuterated form of PAI-1 isolated via the purification protocol was immediately dialyzed into PBS buffer [140 mM NaCl, $3 \mathrm{mM} \mathrm{KCl}, 10 \mathrm{mM}$ $\mathrm{Na}_{2} \mathrm{HPO}_{4}$, and $\left.2 \mathrm{mM} \mathrm{KH}_{2} \mathrm{PO}_{4}(\mathrm{pH} 7.4)\right]$ in $\mathrm{D}_{2} \mathrm{O}$ and then frozen at $-80{ }^{\circ} \mathrm{C}$. Purification was confirmed by SDS-PAGE, accompanied by Western blotting, and MALDI-TOF-MS.

Circular Dichroism (CD). Circular dichroism spectra were measured at $20^{\circ} \mathrm{C}$ on samples in PBS buffer using a model 202 Aviv circular dichroism spectrometer using $1 \mathrm{~mm}$ path-length quartz cuvettes. The online tool Dichroweb was used to analyze data to calculate secondary structure content. ${ }^{50,51}$ Buffer background scans included the same weight percent of PEG-400 and EG and were subtracted from the corresponding protein sample scans.

Small-Angle Neutron Scattering (SANS). SANS experiments were conducted at $20{ }^{\circ} \mathrm{C}$ on protein samples in PBS buffer on the Bio-SANS (CG-3) beamline at the High Flux Isotope Reactor (HFIR) and the EQ-SANS (BL-6) beamline at the Spallation Neutron Source (SNS), both located at Oak Ridge National Laboratory (ORNL). SANS studies using osmolytes were conducted on Bio-SANS using neutrons at a wavelength $\lambda$ of $6.0 \AA$ with a wavelength spread $(\Delta \lambda / \lambda)$ of 0.14 and sample-to-detector distances of 1.2 and $6.9 \mathrm{~m} .{ }^{52}$ SANS contrast variation studies were conducted on EQ-SANS using the $30 \mathrm{~Hz}$ mode and a $4 \mathrm{~m}$ sample-to-detector distance to achieve 2.5-6.1 and 9.4-13.1 $\AA$ wavelength bands. ${ }^{53}$ The wavevector transfer is $q=4 \pi \sin (\theta) / \lambda$, where $2 \theta$ is the 
scattering angle. Circular quartz cuvettes (Hellma USA, Plainville, NY) with a $1 \mathrm{~mm}$ path length were used for SANS measurements on protein samples. Standard procedures for data analysis employed MantidPlot. ${ }^{54}$ The observed scattering intensity was mathematically corrected for detector sensitivity and the measured scattering contribution from the solvent and empty cells. The corrected scattering intensity was then adjusted to an absolute scale using a calibrated standard. ${ }^{55}$

SANS Contrast Variation Measurements. SANS contrast variation was performed on the PAI-1:SMB-IDD complex using $60 \%$ deuterated W175F PAI-1 and protiated SMB-IDD at various $\mathrm{D}_{2} \mathrm{O}$ buffer percentages $(0 \%, 20 \%, 85 \%$, and $100 \%$ $\left.\mathrm{D}_{2} \mathrm{O}\right)$. The sample concentration was $7.7 \mathrm{mg} / \mathrm{mL}$ in all cases. Figure S4 shows a plot of the neutron scattering-length density ( $\mathrm{SLD}$, often written as $\rho$ ) versus the percent of $\mathrm{D}_{2} \mathrm{O}$ in the solvent for water (black line), a protiated protein such as SMBIDD (blue line) and a $60 \%$ deuterated protein (red line) such as PAI- 1 . The plot shows that the SLD of water varies from $-0.56 \times 10^{10} \mathrm{~cm}^{-2}$ for $0 \% \mathrm{D}_{2} \mathrm{O}$, i.e., $\mathrm{H}_{2} \mathrm{O}$, to $6.4 \times 10^{10} \mathrm{~cm}^{-2}$ for $100 \% \mathrm{D}_{2} \mathrm{O}$. The slopes of the lines for the proteins are not as steep as that for water, so the protein and water lines cross each other. Those points are called match points, because the SLDs of protein and water are the same. Thus, the contrast between protein and water (two-sided black arrow shown at $20 \% \mathrm{D}_{2} \mathrm{O}$ in the figure), often written as $\Delta \rho$, is zero at these match points, meaning that the scattering intensity of protein is zero under these conditions. The match point between $60 \%$ deuterated protein and water occurs at $85 \% \mathrm{D}_{2} \mathrm{O}$, as illustrated by the green circle in the figure. This means that, for a complex consisting of protiated protein and $60 \%$ deuterated protein, the scattering from the latter is matched out in $85 \% \mathrm{D}_{2} \mathrm{O}$ buffer, and the only component scattering is the protiated protein, which in this case is the SMB-IDD. In $20 \% \mathrm{D}_{2} \mathrm{O}$ buffer, neither component is matched out. However, the contrast between $60 \%$ deuterated protein and water is larger than that between protiated protein and water. This is true to an even greater extent in $0 \% \mathrm{D}_{2} \mathrm{O}$ buffer. Thus, the scattering from $60 \%$ deuterated PAI-1 dominates the total scattering from the complex in $0 \%$ and $20 \% \mathrm{D}_{2} \mathrm{O}$ buffers but is completely absent in $85 \% \mathrm{D}_{2} \mathrm{O}$ buffer and contributes weakly in $100 \% \mathrm{D}_{2} \mathrm{O}$ buffer.

SANS measurements with osmolytes used protiated SMBIDD in the presence of varying concentrations of deuterated ethylene glycol (EG) (Cambridge Isotope Laboratories, $\mathrm{C}_{2} \mathrm{D}_{6} \mathrm{O}_{2}$ ). Deuterated EG has a match point at $100 \% \mathrm{D}_{2} \mathrm{O}$. Thus, the measurements were performed in $100 \% \mathrm{D}_{2} \mathrm{O}$ buffer to match out deuterated EG so that only the contribution from SMB-IDD is being measured.

The Guinier approximation ${ }^{56}$

$$
I(q)=I(0) \exp \left(-q^{2} \frac{R_{\mathrm{g}}{ }^{2}}{3}\right)
$$

was applied to the low- $q$ region of the data to yield values for $R_{\mathrm{g}}$ and $I(0)$. This approach is valid only for the data region where $Q R_{\mathrm{g}} \lesssim 1.3 R_{\mathrm{g}}$. Taking the natural log of the Guinier approximation (eq 1 ) results in

$$
\ln [I(q)]=\ln [I(0)]-q^{2} \frac{R_{\mathrm{g}}{ }^{2}}{3}
$$

$R_{\mathrm{g}}$ is obtained from the slope of a linear fit to $\ln [I(q)]$ versus $q^{2}$ (eq 2), and $I(0)$ is obtained from the intercept.
$I(0)$ can be expressed as a function of the contrast $\Delta \rho$ as

$$
I(0)=n(\Delta \rho)^{2} V^{2}
$$

where $n$ is the number density of molecules in solution, which is proportional to the sample concentration, and $V$ is the molecular volume. Because $I(0)$ is proportional to $c$ and $\Delta \rho^{2}$, and $\Delta \rho^{2}$ is dependent on the percent of $\mathrm{D}_{2} \mathrm{O}$ in the solvent, the $x$-intercept of a linear fit to $\sqrt{I(0) / c}$ versus percent $\mathrm{D}_{2} \mathrm{O}$ in the solvent is at the contrast match point of the complex. If the sample concentrations are the same at all contrasts measured, then $\sqrt{I(0)}$ versus percent $\mathrm{D}_{2} \mathrm{O}$ can be used instead. The quality of the data can be tested by also calculating the match point of the complex from the amino acid sequences of the components. ${ }^{57,58}$ If the complex was formed as expected, the calculated and experimentally determined match points should agree with each other.

Analysis of SANS Data using Stuhrmann Analysis and the Parallel Axis Theorem. The $R_{\mathrm{g}}$ values obtained from the data at each contrast can be described by the equation ${ }^{59}$

$$
R_{\mathrm{g}}{ }^{2}=R_{\mathrm{m}}{ }^{2}+\frac{\alpha}{\Delta \rho}-\frac{\beta}{\Delta \rho^{2}}
$$

where $R_{\mathrm{m}}$ is the $R_{\mathrm{g}}$ value of the equivalent complex with a homogeneous scattering-length density, $\alpha$ is the second moment of the SLD fluctuations, and $\beta$ is the square of the first moment of the SLD fluctuations. For two-component systems with different SLDs such as protiated SMB-IDD and $60 \%$ deuterated PAI- $1, \alpha$ describes the distribution of SLDs relative to the center of mass (CM) of the complex, and $\beta$ is related to the separation distance between the scattering $\mathrm{CM}$ of the two components (CM distance). ${ }^{60}$ A Stuhrmann plot ${ }^{59}$ of $R_{\mathrm{g}}{ }^{2}$ versus $\Delta \rho^{-1}$ (from eq 4 ) is used to determine $R_{\mathrm{m}}, \alpha$, and $\beta$. If the plot is linear $(\beta=0)$, the CMs of the two components are concentric and the sign of the slope, $\alpha$, indicates whether the component with the higher SLD is on the interior or exterior of the complex.

A related equation is the parallel axis theorem

$$
R_{\mathrm{g}}{ }^{2}=f_{1} R_{1}{ }^{2}+f_{2} R_{2}{ }^{2}+f_{1} f_{2} D_{\mathrm{CM}}{ }^{2}
$$

where $R_{1}$ and $R_{2}$ are the radii of gyration of the components and $D_{\mathrm{CM}}$ is the CM distance between the two components. ${ }^{61}$ Here

$$
f_{i}=\frac{\Delta \rho_{i} V_{i}}{\Delta \rho V}
$$

where $\Delta \rho_{i}$ and $V_{i}$ refer to components 1 and 2 and $\Delta \rho$ and $V$ refer to the entire complex. The radii of gyration of the components and the CM distance between them are obtained from the parallel axis theorem directly, whereas these parameters are calculated from the definitions of $\alpha$ and $\beta$ when using the Stuhrmann analysis. ${ }^{57}$ Both analyses were performed on the PAI-1:SMB-IDD contrast variation data using the MULCh software. ${ }^{57}$ The $0 \%, 20 \%$, and $85 \% \mathrm{D}_{2} \mathrm{O}$ conditions were used in the analyses.

Analysis of SANS Data using EOM. The SMB-IDD structure, with and without added osmolytes, was assessed using the EOM program within the ATSAS program suite. ${ }^{62}$ The same software was used to determine the flexibility of the unbound and PAI-1-bound forms of SMB-IDD. Known sequence and structural information for the SMB-IDD served as a basis to generate 10000 independent structures using 
default EOM settings. ${ }^{63-66}$ EOM-calculated scattering curves of these structures were judged in comparison to the experimental SANS data using a genetic algorithm to select 1000 structures that average to best fit the SANS measurements. The results are represented as a distribution in $R_{\mathrm{g}}$. For the complex, the SANS data collected on the deuterated PAI1:SMB-IDD complex in $100 \% \mathrm{D}_{2} \mathrm{O}$ were used, where the SMB-IDD signal is dominant. The Protein Data Bank (PDB) coordinates for SMB-IDD were extracted from the selected EOM structures, and the $R_{\mathrm{g}}$ of the ensemble (SMB-IDD only) was calculated using CRYSOL $^{67}$ to directly compare with the $R_{\mathrm{g}}$ distribution from unbound SMB-IDD.

Analysis of SANS Data using SASSIE. SASSIE ${ }^{68}$ was developed at the National Institute of Standards and Technology (NIST) as a suite of software tools for rendering high-resolution representations of protein structures and/or complexes that fit to the corresponding SANS data. The beginning point in generating these models was energy minimization to produce "starting structures" with all-atom atomic coordinates using the co-crystal structure for the PAI$1: S M B$ complex (PDB entry 1OC0 $)^{49}$ as the basis data set. Several amino acid replacements were modeled into this structure, including the $\mathrm{W} 175 \mathrm{~F}$ point mutation, the five residues missing from the $\mathrm{N}$-terminus, and the short segment of the reactive center loop (RCL) that is not resolved in the crystal structure. NAMD was used for energy minimization of the RCL for 1000 steps. ${ }^{69}$ Subsequently, energy minimization of the rebuilt PAI-1 structure was implemented for an additional 1000 steps. Torsion-angle molecular dynamics ${ }^{70}$ (10 ps) were applied to the RCL portion of the resulting minimized PAI-1 structure; the final frame was adopted as the PAI-1 component within each of the calculated starting structures.

Starting Structure 1. For all three starting structures, the SMB domain within the SMB-IDD was generated from the three-dimensional coordinates for the SMB structure in the cocrystal (PDB entry 1OC0), which defined the PAI-1:VN interface. A segment of 10 disordered residues was added to the known SMB structure, based on data from the published nuclear magnetic resonance (NMR) structure. ${ }^{71}$ For starting structure 1 , the additional residues up to the end of the IDD sequence were added and allowed to be flexible, absent any constraints in the energy minimization. NAMD was used to minimize the SMB-IDD structure for 1000 steps. Finally, the separately minimized PAI-1 and VN structures were built back into a single model for an additional 1000 steps of energy minimization to produce starting structure 1 .

Starting Structure 2. For the second structure, a similar approach was taken as with construction of starting structure 1, but structural constraints were imposed on the IDD segment. Specifically, a compact structure for the IDD portion of starting structure 2 was desired, for better agreement with the experimentally determined $R_{\mathrm{g}}$. PSIPRED, PHYRE2, ChouFasman, GOR, and neural network were used ${ }^{72-77}$ to predict helical regions and seek agreement from more than one method. Even though some algorithms predicted short helical regions, no segment of the IDD was consistently predicted as helical comparing these methods, so none was introduced. Nonetheless, PSIPRED yielded a more compact structure for the IDD, which was incorporated into the model rather than the extended IDD without constraints in starting structure 1 . Energy minimization of this model for 5000 steps yielded starting structure 2 .
Starting Structure 3. Starting structure 3 incorporated additional constraints to produce a starting structure that had a CM distance between the two components $(20.8 \AA)$ that was more comparable to that calculated from the experimental data $[18 \pm 1 \AA$ (see Table S2) $]$. Force was applied to the IDD from starting structure 2 via the interactive molecular dynamics (MD) option within Visual Molecular Dynamics ${ }^{78}$ to orient the IDD on the opposite side of PAI-1, lying across the central $\beta$-sheet. Energy minimization of this model for 5000 steps yielded starting structure 3 .

From each of these starting structures, 50000 additional structures were generated. The Complex Monte Carlo module in SASSIE was used to vary the disordered portion of the $\mathrm{VN}$ component, generating ensembles of structures from the three starting PAI-1:SMB-IDD structures for comparison to SANS data. Configurations were generated by sampling backbone dihedral angles using CHARMM-27 all-atom protein force field parameters. ${ }^{79}$ Each new configuration was checked for overlap of $\alpha$-carbon atoms. If the overlap distance between these atoms was $\geq 3 \AA$, the new structure was accepted. To eliminate structures with correlated dihedral angle moves, only every 10th accepted structure was selected for further analysis. The final ensembles consisted of 3000-5000 accepted structures. A second Complex Monte Carlo run was performed from starting structures 1 and 3 in which $R_{\mathrm{g}}$ was constrained to values of $<35 \AA$ to obtain more structures with $R_{\mathrm{g}}$ values close to that obtained from the data. These final ensembles contained approximately 2000 structures.

The $0 \%, 20 \%$, and $85 \% \mathrm{D}_{2} \mathrm{O}$ samples were used in the structure modeling analysis of the PAI-1:SMB-IDD complex. SANS curves were calculated for the ensembles of PAI-1:SMBIDD model structures with the SasCalc ${ }^{80}$ module in SASSIE, using the converged number of golden vectors option and a tolerance of 0.001 . A H-D exchange fraction of 0.95 was used for the PAI-1 and VN components, and the PAI-1 component was assumed to be $60 \%$ deuterated. For SMB-IDD alone in solution, a H-D exchange fraction of 0.95 was used.

The calculated SANS curves were compared to data using the $\chi^{2}$ filter module in SASSIE. The $\chi^{2}$ equation was used to assess the goodness of fit to the data.

$$
\chi^{2}=\frac{1}{N-1} \sum_{q} \frac{\left[I_{\exp }(q)-I_{\text {calc }}(q)\right]^{2}}{\sigma_{\exp }(q)^{2}}
$$

where $I_{\exp }(q)$ is the experimental SANS intensity curve, $I_{\text {calc }}(q)$ is the calculated SANS intensity curve from the model structure, and $\sigma_{\exp }(q)$ is the $q$-dependent error of the $I_{\exp }(q)$ values. The sum was taken over 51 data points $(N)$ in this case.

A $\chi^{2}$ versus $R_{\mathrm{g}}$ analysis provided a measure of how well the individual structures generated from each starting structure fit the data and which starting structure resulted in the best fits to the data. Structure ensembles were further evaluated on the basis of the shapes of the $\chi^{2}$ versus $R_{\mathrm{g}}$ plots to find the best fit ensemble of structures. Surface density plots showing the range of conformational space covered by the IDD region were made using the Density Plot module of SASSIE. This provided a visual comparison of the conformational space spanned by SMB-IDD when bound to PAI-1 and when free in solution.

\section{RESULTS}

Computational Predictions of the Intrinsically Disordered Nature and Potential of the IDD To Adopt Structure. In line with the considerations discussed in the 
a

b
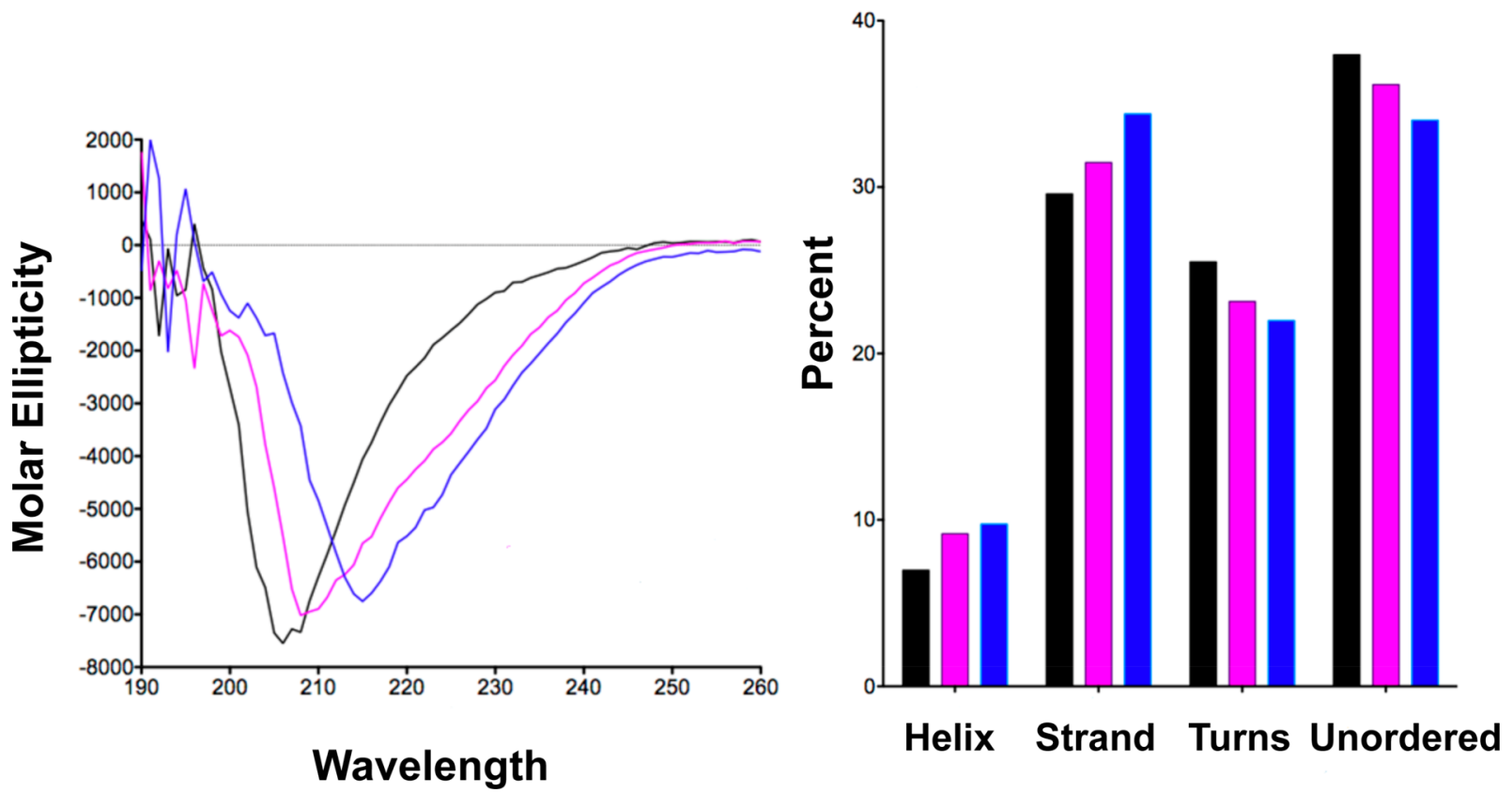

Figure 2. Effects of osmotic pressure on the secondary structure of the SMB-IDD fragment of VN. (a) CD spectra collected at various concentrations of osmolytes: black line, isolated SMB-IDD; pink line, SMB-IDD with 57\% EG added; blue line, SMB-IDD with 57\% PEG-400 added. (b) Secondary structure components calculated from the CD data, signifying a decrease in the level of disorder and an increase in the level of structure with the IDD of VN under osmotic pressure: black, isolated SMB-IDD; pink, SMB-IDD with 57\% EG added; blue, SMB-IDD with 57\% PEG-400 added.

introductory section, Figure 1a shows the results of the multiparametric evaluation of the predisposition for intrinsic disorder across the entire sequence of human vitronectin (UniProt ID P04004) employing several frequently used disorder predictors, including PONDRVLXT, ${ }^{81}$ PONDR VSL2, ${ }^{82}$ PONDR VL3, ${ }^{82,83}$ PONDR FIT, ${ }^{84}$ IUPred_short, and IUPred_long. ${ }^{85,86}$ The rationale for the selection of these tools is provided in previous publications. ${ }^{87,88}$ Also, the average of the outputs of these six per-residue disorder predictors was used to calculate a mean per-residue intrinsic disorder profile for vitronectin. The use of consensus or mean disorder propensity is consistent with empirical observations, indicating that this strategy typically increases the predictive performance compared to the use of a single predictor. ${ }^{89-91}$ The individual computational tools and consensus disorder propensity in Figure 1a uniformly indicate that the IDD from vitronectin is predicted to be highly disordered.

A hallmark feature of many intrinsically disordered domains is their ability to adopt structure upon interaction with a binding partner. To test the potential of the IDD of VN to behave similarly, we analyzed the IDD sequence using the Molecular Recognition Feature (MoRF) algorithm. ${ }^{12}$ Within intrinsically disordered proteins, MoRFs are segments that are predicted to undergo a change from disorder to order when bound to a ligand. The ordered conformation acquired by the protein varies and could include adoption of secondary structure or folding to an ordered loop structure. ${ }^{92}$ The results of the MoRF prediction for the vitronectin IDD are shown in Figure $1 \mathrm{~b}$, identifying two segments within the IDD (VN residues 52-72 and 84-95) that may adopt $\alpha$-helical structure in a coupled folding-binding event upon interaction with a ligand. These predictions bolster the hypothesis that binding to PAI-1 leads to a change in the conformation of the IDD of VN.

Using Circular Dichroism To Study the Effect of Osmolytes on the Vitronectin IDD Structure. In the pursuit to determine whether this interdomain sequence (amino acids 48-130) from VN exhibited properties expected for an IDD region, we evaluated the ways in which osmolytes affect the structure of the IDD. Osmotic pressure, which perturbs the solvation of a protein, is frequently used to interrogate folding propensity and reveal structural changes that could be obscured in the presence of binding partners. ${ }^{93-95}$ In particular, osmolytes have been especially useful in studies to demonstrate folding in intrinsically disordered proteins, due to the crowding effect that emulates cellular conditions and confers compaction and adoption of structure in predisposed regions. ${ }^{96-99}$

We utilized far-ultraviolet (far-UV) CD to study the effect of osmotic pressure on secondary structure within the IDD. Ethylene glycol (EG) and polyethylene glycol (PEG) 400 were employed to test for structural changes within the IDD that may be induced by changes in osmotic pressure. The results indicated that the SMB-IDD fragment alone exhibited ellipticity characteristic of a protein fold devoid of classical secondary structure elements (Figure 2a). However, upon addition of either osmolyte, we observed the ellipticity measured for SMB-IDD shifted toward $220 \mathrm{~nm}$, indicating a reduction in the level of disorder and a gain of some ordered secondary structure (Figure 2a). The two different osmolytes, EG and PEG-400, were used to assess any osmolyte size effects. For the same weight percent, PEG-400 has an osmotic pressure that is lower than that of EG. However, PEG-400 induced more secondary structure in SMB-IDD compared to 
a

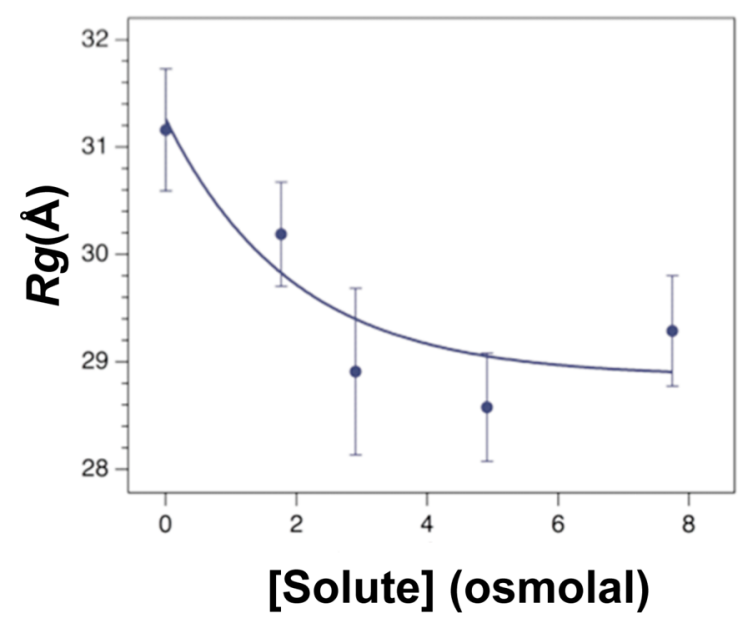

b

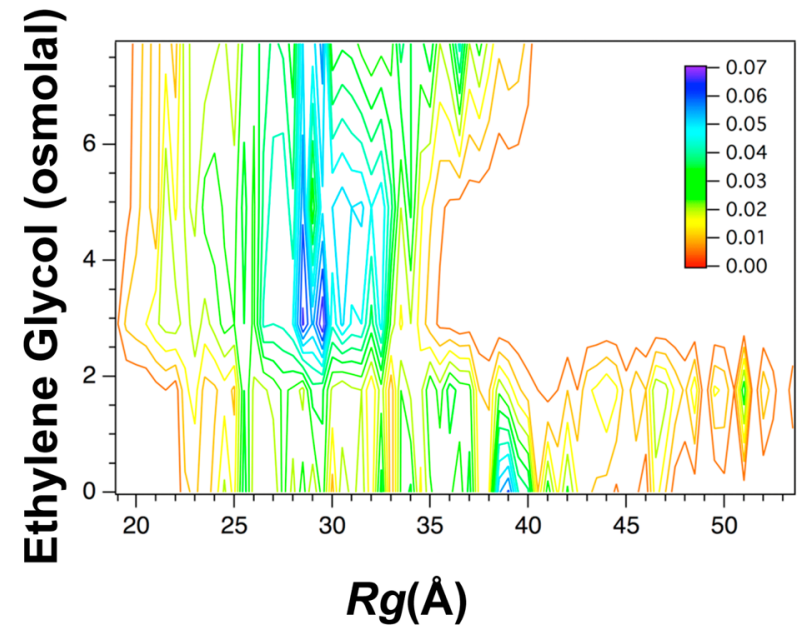

Figure 3. (a) Effect of osmotic pressure on the structure of the SMB-IDD measured by SANS. $R_{\mathrm{g}}$ values from SANS measurements on SMB-IDD in $100 \% \mathrm{D}_{2} \mathrm{O}$ with addition of deuterated EG are plotted vs osmolyte concentration. Error bars represent the standard deviation. (b) Heat map from EOM analysis to illustrate effects of osmotic pressure on the size of the SMB-IDD. The SMB-IDD $R_{\mathrm{g}}$ distribution $(x$-axis, where the color bar legend represents the relative frequency) is observed to shift to a lower range and narrow as osmotic stress is applied with deuterated EG (plotted along the $y$-axis). Notably, the most prevalent $R_{\mathrm{g}}$ (dark blue) in the absence of osmolyte, which is $\sim 39 \AA$, decreases by $\sim 10 \AA$ over the course of EG addition, confirming that osmolytes perturb the structure of the IDD.

EG due to a larger excluded volume. A comparison of these results shows that more than one osmolyte induces effects on SMB-IDD structure, pointing toward general action rather than osmolyte-specific effects. The far-UV CD results were analyzed to identify changes in secondary structure with the web-based tool, Dichroweb, which quantifies $\alpha$ - and $\beta$ structures and loops. The CD experiments corroborated our hypothesis that osmolytes can induce a disorder-to-order transition in the IDD (Figure $2 \mathrm{~b}$ ).

Determining the Effect of Osmolytes on the IDD Structure Using Small-Angle Neutron Scattering (SANS). The manner in which osmolytes perturb the structure of the SMB-IDD was also investigated by SANS. Using deuterated EG in a $100 \% \mathrm{D}_{2} \mathrm{O}$ buffer to observe only protein signal, SANS showed that addition of EG led to a smaller $R_{\mathrm{g}}$ for SMB-IDD (Figure S1 and Figure 3a). This decrease in $R_{\mathrm{g}}$ indicated that the solution structure for the IDD became more compact with an increase in osmotic stress. EOM was employed to provide additional in-depth analysis of the SANS data. As described in Materials and Methods for this approach, 10000 possible structures for the SMB-IDD were generated, along with their corresponding scattering curves to be compared with the measured SANS data. From the 10000 structures, the best fitting 1000 were identified for each of the osmolyte conditions in Figure $3 \mathrm{a}$. Using the selected structures, a heat map was generated comparing the $R_{\mathrm{g}}$ distribution as a function of ethylene glycol concentration (Figure $3 \mathrm{~b}$ ). This analysis indicates that the isolated SMB-IDD is a significantly flexible structure, existing over a relatively broad $R_{\mathrm{g}}$ range of 20-55 $\AA$, but sampling a significantly smaller range when under osmotic stress (Figure $S 1$ and Figure $3 b$ ). This structural transition, revealed by a smaller $R_{\mathrm{g}}$ range, could correspond to an increase in the level of secondary structure and/or an overall compaction of the IDD. While the limited resolution of small-angle scattering approaches is insufficient to distinguish these two possibilities, the corresponding results from $\mathrm{CD}$ indicated that some gain in secondary structure occurred.
Evaluating Changes in the IDD Structure That Occur upon PAI-1 Binding Using Contrast Variation Measurements with SANS. Taken together, the results of assays with osmolytes suggest that the IDD within vitronectin acquires secondary structure as observed for many intrinsically disordered proteins. ${ }^{93-99}$ These findings provide a rationale for evaluating the association of PAI-1 with the vitronectin fragment (SMB-IDD) to determine whether binding of a cognate ligand also perturbs the folding of the IDD. For these experiments, W175F PAI-1, deuterated to a final level of $60 \%$, was produced to be used in combination with protiated SMBIDD in the measurements of the PAI-1:SMB-IDD complex to differentiate scattering data between the two complex components via contrast variation experiments. For the contrast variation experimental design, the level of $\mathrm{D}_{2} \mathrm{O}$ in the solution was varied so that the scattering signal from one of the two proteins predominated under the varied solvent compositions, thus exploiting the change in scattering that occurs when deuterium is present. Collection of data at multiple buffer $\mathrm{D}_{2} \mathrm{O}$ percentages allows for isolation and/or suppression of components within the complex from the scattering data as explained in Materials and Methods, so that a model depicting the arrangement of the proteins within the complex can be assembled. SANS data at four different levels of deuterated solvent are shown in Figure S2. Corresponding Guinier plots (eqs 1 and 2) are shown in Figure S3, and $R_{\mathrm{g}}$ and $I(0)$ values for the data sets are listed in Table S1. Briefly, the $R_{\mathrm{g}}$ values obtained for the data in $0 \%$ and $20 \% \mathrm{D}_{2} \mathrm{O}$ were similar $(25.45 \pm 0.07$ and $24.95 \pm 0.09 \AA$, respectively), whereas the value for $85 \% \mathrm{D}_{2} \mathrm{O}$ is higher, at $28 \pm 3 \AA$. Finally, $R_{\mathrm{g}}$ in $100 \% \mathrm{D}_{2} \mathrm{O}$ was found to be $31.3 \pm 0.4 \AA$. Note that the errors on the $0 \%$ and $20 \% \mathrm{D}_{2} \mathrm{O}$ values are the standard errors from the linear fit to the Guinier equation. They are approximately an order of magnitude smaller than the sensitivity of the SANS technique under the best signal-tonoise conditions. On the other hand, the error on the $85 \%$ $\mathrm{D}_{2} \mathrm{O}$ value was taken from an average value obtained from two 
Guinier fits in slightly different $q$ ranges, as the data were noisy because only the VN component is scattering under these conditions. Thus, the error is more in line with the sensitivity of the SANS technique for measurements made under poor signal-to-noise conditions. Finally, the error on the $100 \% \mathrm{D}_{2} \mathrm{O}$ value is in line with the sensitivity of the SANS technique under the best signal-to-noise conditions.

A plot of $\sqrt{I(0)}$ versus percent $\mathrm{D}_{2} \mathrm{O}$ in the solvent (eq 3 ) is shown in Figure S5. The $x$-intercept from a weighted linear fit (solid line) to the data yielded a match point of $76.29 \pm 0.03 \%$ $\mathrm{D}_{2} \mathrm{O}$. Because all samples were at the same concentration $(7.7$ $\mathrm{mg} / \mathrm{mL}$ ), it was not necessary to divide $I(0)$ by the concentration to determine the match point. The match point for the PAI-1:SMB-IDD complex obtained from the amino acid sequences of the components was determined to be $77 \% \mathrm{D}_{2} \mathrm{O}$ using both $\mathrm{MulCh}^{57}$ and the Contrast Calculator module in SASSIE, ${ }^{68}$ assuming a 1:1 PAI-1:SMB-IDD complex with PAI-1 $60 \%$ deuterated. The agreement of the experimental and calculated match point verifies that PAI-1 is $60 \%$ deuterated as expected. Thus, PAI- 1 was assumed to be $60 \%$ deuterated in a 1:1 PAI-1:VN complex for the Stuhrmann and parallel axis theorem analyses as well as for calculation of model SANS curves.

The results from the Stuhrmann and parallel axis theorem analyses (eqs 4-6) of the $0 \%, 20 \%$, and $85 \% \mathrm{D}_{2} \mathrm{O}$ data are shown in Table S2. $R_{\mathrm{g}}=23.6 \pm 0.1 \AA$ for PAI-1, and $R_{\mathrm{g}}=30.3$ $\pm 0.3 \AA$ for SMB-IDD in the complex. The CM distance between the two components is $18 \pm 1 \AA$. In addition, the $\alpha$ parameter from the Stuhrmann analysis was negative, indicating that the component with the lower scattering-length density (protiated SMB-IDD) lies toward the periphery of the complex. Taken together, these results suggest that the IDD is compact and located close to the PAI-1 component.

The $100 \% \mathrm{D}_{2} \mathrm{O}$ data were not used in the final Stuhrmann and parallel axis theorem analyses or the structure modeling because a valid result with positive $R_{\mathrm{g}}{ }^{2}$ values in eqs 4 and 5 could not be obtained when these data were included. It is very possible that there may be a small amount of $\mathrm{D}_{2} \mathrm{O}$-induced aggregation under these solvent conditions. The experimental value for PAI-1 in the complex is somewhat larger than the $R_{\mathrm{g}}$ value for PAI- 1 of $21.2 \AA$ calculated from the crystal structure of isolated PAI- $1^{49}$ or $21.86 \AA$ calculated for the PAI-1 component in our PAI-1:SMB-IDD starting structures. While MD simulations could be performed on the PAI-1 component in the complex to obtain a potential $R_{\mathrm{g}}$ value that is closer to the experimental value, it was not found to be necessary to obtain good fits to the $0 \%$ and $20 \% \mathrm{D}_{2} \mathrm{O}$ data.

For data analysis, we once again used EOM to provide insight into whether the SMB-IDD adopted a more compact structure in the presence of the PAI-1 ligand. As described in Materials and Methods, we were able to determine the individual contribution from the SMB-IDD in the PAI-1bound state. We compared the $R_{\mathrm{g}}$ values for free versus bound SMB-IDD and observed that PAI-1 binding yields a shift in the $R_{\mathrm{g}}$ range for the SMB-IDD to predominantly lower values, consistent with a solution structure for the IDD that is restricted in conformation upon binding (Figure 4). An important question is the stoichiometry of the complexes measured in this study, which cannot be assumed and must be measured in the SANS work. Our prior work using hydrodynamic methods with PAI-1 and full-length vitronectin demonstrated that there is a $2: 1$ (PAI-1:vitronectin)

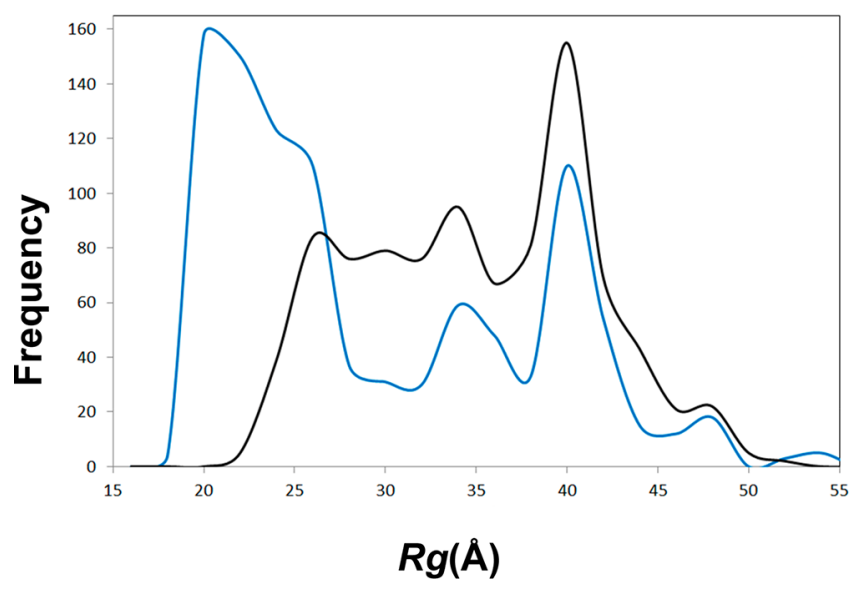

Figure 4. Comparison of $R_{\mathrm{g}}$ values from analysis by EOM for SANS data on the PAI-1:SMB-IDD complex vs unbound SMB-IDD. Evaluation of scattering curves by EOM for unbound SMB-IDD indicates that the IDD samples a broad range of conformational space. A comparison of the SANS data for unbound (black) vs bound (blue) illustrates the shift in the $R_{\mathrm{g}}$ across the range upon PAI-1 binding, corresponding to a predominance of conformations with a decreased $R_{\mathrm{g}}$ when the ligand is bound.

stoichiometry of interaction that leads to assembly of higherorder oligomeric complexes via a prominent intermediate containing four PAI-1 and two vitronectin molecules. ${ }^{100}$ Thus, it was not clear whether we would observe a $2: 1$ or $1: 1$ interaction for PAI-1 with the truncated form of vitronectin (SMB-IDD). The $I(0)$ values calculated from the amino acid sequences of the $60 \%$ deuterated PAI- 1 and $\mathrm{VN}$ in $0 \%, 20 \%$, and $85 \% \mathrm{D}_{2} \mathrm{O}$, assuming a $1: 1$ complex at a concentration of $7.7 \mathrm{mg} / \mathrm{mL}$, agreed with those obtained from the measured SANS data (Figure S2 and Table S1), indicating that the complex is $1: 1$ at these contrasts. This is supported by our recent study using stopped-flow kinetics to characterize biphasic binding of PAI-1 with SMB-IDD versus monophasic binding with SMB. ${ }^{46}$ The biphasic binding observed corroborates the binding of the IDD to PAI-1, with separate binding rates for the SMB and IDD regions; this prior work also demonstrated a 1:1 binding between these proteins. ${ }^{46}$

Structural Modeling of the SMB-IDD from SANS Analysis. To generate reasonable models for the SMB-IDD, free and bound to the PAI-1 ligand, we generated a large set of possible structures and their corresponding scattering patterns to compare with the measured SANS data. These calculations utilized the SASSIE software suite, as described in Materials and Methods. The analysis begins by generating starting structures and using Monte Carlo and MD approaches to explore protein conformations that are consistent with the measured SANS data. The starting structures are shown in Figure 5. Briefly, starting structure 1 was created without constraints on the IDD; starting structure 2 was produced with $R_{\mathrm{g}}$ constraints on the IDD that yield a more compact structure that better matched the experimental data, and starting structure 3 was generated by constraining $R_{\mathrm{g}}$ and forcing a closer approximation of the IDD and PAI-1 (i.e., the IDD "wrapped around" PAI-1) to better align with the CM distance calculated from the SANS data. Also, a starting structure for the unbound SMB-IDD was calculated without constraints on the IDD (Figure 5). More details regarding the generation of the starting structures are provided in Materials and Methods. 


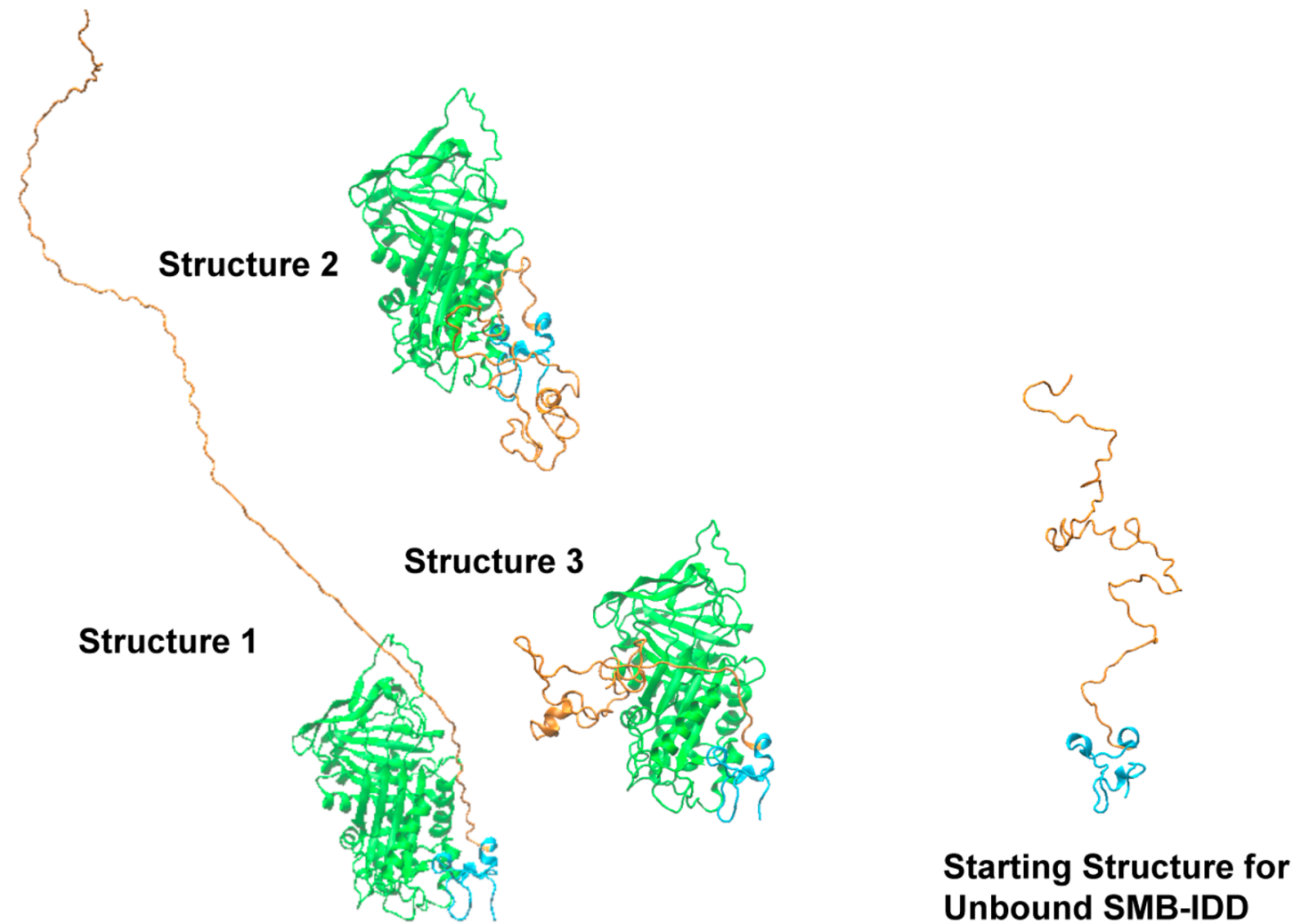

Starting Structures for SMB-IDD Bound to PAI-1

Figure 5. Starting structures for evaluating SANS data on the PAI-1:SMB-IDD complex or unbound SMB-IDD. Starting structures representing unbound SMB-IDD or the PAI-1:SMB-IDD complex were generated as basis data sets for employing Monte Carlo and molecular dynamics methods to calculate sizable, conformationally diverse pools of structures for data analysis. The three starting structures for SMB-IDD bound to PAI-1 were produced with the all-atom calculator within the SASSIE tool suite. Structure 1 lacked constraints on the IDD. Structure 2 was calculated with the IDD in a more compact conformation. Structure 3 was generated with the compact IDD conformation in close proximity to PAI-1. A single starting structure for the free SMB-IDD was used for analysis of the corresponding SANS data. PAI-1 is colored green, the SMB domain cyan, and the IDD amber.

From each of the three starting structures for the SMB-IDD complex (Figure 5), pools of 3000-5000 accepted (nonoverlapping) conformations were generated as described in Materials and Methods. Calculated scattering data for these pools of structures were compared to the experimental SANS data, and $\chi^{2}$ values (eq 7) were assessed as a measure of the goodness of fit. The $\chi^{2}$ versus $R_{\mathrm{g}}$ values for the three individual structures are compared for the $85 \% \mathrm{D}_{2} \mathrm{O}$ contrast solution in Figure $6 \mathrm{a}$. The $85 \% \mathrm{D}_{2} \mathrm{O}$ contrast was selected because PAI-1 with $60 \%$ deuteration is matched out under these conditions (match point of $85 \% \mathrm{D}_{2} \mathrm{O}$ as shown in Figure S4), so that SMB-IDD dominates the measurement in this case. The best fit to the measurements in $85 \% \mathrm{D}_{2} \mathrm{O}$ is observed with the two pools arising from starting structure 1 (colored red and black). The ensemble colored red, in which $R_{\mathrm{g}}$ was constrained to $<35$ $\AA$, was further analyzed by calculating $\chi^{2}$ versus $R_{\mathrm{g}}$ at the other two measured contrasts, $0 \%$ and $20 \% \mathrm{D}_{2} \mathrm{O}$. The results are shown in Figure $6 \mathrm{~b}$, where the red points for the $85 \% \mathrm{D}_{2} \mathrm{O}$ data are the same as the red points in Figure $6 \mathrm{a}$, the gray points are for the $20 \% \mathrm{D}_{2} \mathrm{O}$ data, and the purple points are for the $0 \%$ $\mathrm{D}_{2} \mathrm{O}$ data.

Additionally, the $\chi^{2}$ versus $R_{\mathrm{g}}$ correlation for the SANS measurements on the isolated SMB-IDD is plotted in Figure 7. As observed for the PAI-1:SMB-IDD complex, a subset of structures for the unbound SMB-IDD does not provide good fits to the experimental data, although a significant number of structures exhibit reasonable $\chi^{2}$ values.

Density plots were generated to aid in the visualization and comparison of the three-dimensional space accessed by the flexible IDD under both conditions. Figure $8 \mathrm{a}$ presents a density plot representing all structures that fit to the data with a $\chi^{2}$ of $<1.5$ for the free SMB-IDD. The cyan shape in the center of the diagram corresponds to the SMB, and the gray cloud represents the conformational space occupied by the ensemble of calculated structures for the IDD. For comparison, Figure $8 \mathrm{~b}$ provides an overlay of the density plot of the data from SMB-IDD bound to PAI-1 (using starting structure 1) and the density plot for the unbound SMB-IDD. As with panel a, the cyan structure represents the SMB domain, and the green structure in the center represents PAI-1. The amber coloring is a representation of the area of $\chi^{2}<4$ conformational space sampled by the IDD when PAI- 1 is bound to the SMB-IDD, to be compared with the cloud of $\chi^{2}<1.5$ conformational space for the unbound IDD shown in the lighter gray representation. As observed in the overlay in Figure $8 \mathrm{~b}$, the IDD conformations for SMB-IDD bound to PAI-1 inhabit the region surrounding PAI-1 and represent a smaller region of conformational space compared to the unbound SMB-IDD alone. This three-dimensional representation of the free and bound forms of SMB-IDD supports the EOM analysis and expands upon it with a visualization of the 


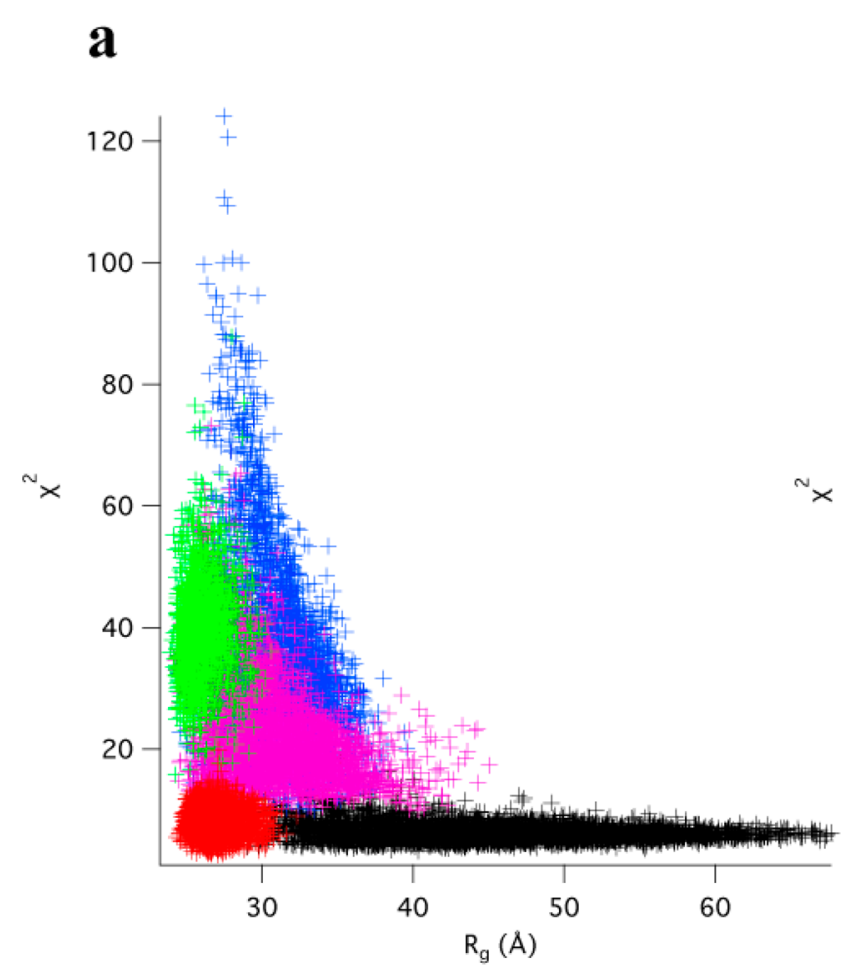

b

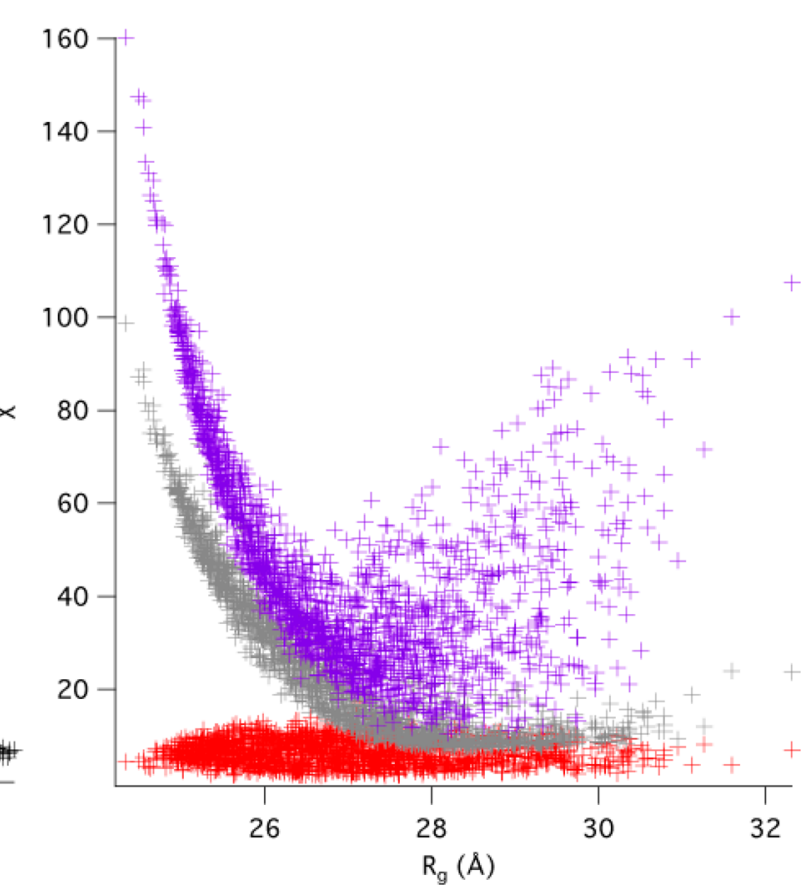

Figure 6. (a) $\chi^{2}$ vs $R_{\mathrm{g}}$ correlation for SANS on PAI- 1 :SMB-IDD in the $85 \% \mathrm{D}_{2} \mathrm{O}$ contrast solution. The starting structures for the complex, shown in Figure 5, were used to calculate large, conformationally diverse pools of structures. Two pools were created from starting structure 1 . One (black) was not subjected to any constraints, while the second pool (red) was restricted to $R_{\mathrm{g}}$ values of $<35 \AA$ A to limit the pool to structures that fit more closely with the experimentally determined $R_{\mathrm{g}}$ values. Starting structure 2 was not subjected to any additional constraints for the generation of its corresponding single pool of structures (blue). Two separate structural pools were also generated from starting structure 3; one (pink) had no additional constraints imposed, and the second (green) was limited to $R_{\mathrm{g}}$ values of $<35 \AA$ and also had constraints imposed to maintain the IDD conformation wrapped around PAI-1. The pools of structures were compared with the SANS data from the $85 \% \mathrm{D}_{2} \mathrm{O}$ condition using $\chi^{2}$ analyses. As one can see from this plot, the two pools generated from starting structure 1 best fit the $85 \%$ contrast data. (b) $\chi^{2}$ vs $R_{\mathrm{g}}$ plot for SANS data on the PAI-1:SMB-IDD complex at all contrasts. The 1797 structures with the lowest $\chi^{2}$ values from starting structure 1 (red data points in panel a) were compared with the SANS measurements at all contrasts for goodness of fit and are depicted in a $\chi^{2}$ vs $R_{\mathrm{g}}$ plot. The red points are from the $85 \%$ $\mathrm{D}_{2} \mathrm{O}$ data and are the same as the red points in panel a. The gray and purple points are from the $20 \%$ and $0 \% \mathrm{D}_{2} \mathrm{O}$ data, respectively.

space to which the IDD is localized under the two conditions. It is noteworthy that starting structure 1 , which has the least conformational restraints of all three starting structures, was used for this density plot, avoiding biasing of conformational space to more compact structures. The agreement of this modeling with the SANS data provides strong support for adoption of a compact structure by the IDD upon interaction with PAI-1. This observed adoption of structure appears not to be simple steric restriction of the IDD region solely due to binding of the SMB domain to PAI-1; comparison of data in Figures 6 and 7 indicates that the SMB-IDD accesses the same lower end of the conformational region $(\sim 20-22 \AA)$ under experimental conditions with and without PAI-1 present.

The calculated SANS curves for the best fit structure $\left(\chi^{2}=\right.$ 1.29 ) for the $85 \% \mathrm{D}_{2} \mathrm{O}$ data (from the ensemble colored red in panels $\mathrm{a}$ and $\mathrm{b}$ of Figure 6) are shown along with the SANS data in $0 \%, 20 \%$, and $85 \% \mathrm{D}_{2} \mathrm{O}$ in Figure 9. Given the flexibility of the IDD, it is unlikely that this single structure exists in solution. Instead, multiple conformations of the IDD likely are present and the average scattering from those structures suggests that the IDD is compact. To explore additional structures that may fit the data as well as the best fit structure, the ensemble of structures represented by the $\chi^{2}$ versus $R_{\mathrm{g}}$ curves in Figure $6 \mathrm{~b}$ was further mined to find the smallest set of structures that best fit the SANS data at all contrasts, while still containing the best fit structure in $85 \%$
$\mathrm{D}_{2} \mathrm{O}$, from which the scattering curves in Figure 9 were calculated. This resulted in 30 structures that fit the SANS data with $\chi^{2}<4$ in $85 \% \mathrm{D}_{2} \mathrm{O}, \chi^{2}<15$ in $20 \% \mathrm{D}_{2} \mathrm{O}$, and $\chi^{2}<20$ in $0 \% \mathrm{D}_{2} \mathrm{O}$, as illustrated in Figure 10. Because all 30 of these structures fit the SANS data at all three contrasts, it follows that linear combinations of these structures can be found that also fit the SANS data at all three contrasts. In addition, the average scattering curve for all of the structures also will fit the SANS data at all three contrasts, meaning that all of the structures in this ensemble could exist in the solution. Furthermore, it is reasonable to assume that additional structures can be found that would also be a good fit to the data at all three contrasts. Table S3 lists the $R_{\mathrm{g}}$ values of PAI-1 and SMB-IDD in the complex, along with the CM distance between them, for the 30 best fit structures at all contrasts from Figure 10. $R_{\mathrm{g}}$ for PAI-1 is fixed at $21.86 \AA$, but that for SMB-IDD varies between 28 and $38 \AA$. CM distances range from 20 to $33 \AA$. In addition, the CM distances and $R_{\mathrm{g}}$ of SMBIDD are coupled in the sense that those structures with larger $R_{\mathrm{g}}$ values for SMB-IDD have smaller CM distances. The best fit structure (number 16) has an $R_{\mathrm{g}}$ of SMB-IDD (31.0 $\AA$ ), very close to that predicted from the Stuhrmann and parallel axis theorem analyses $(30.3 \pm 0.3 \AA)$. However, the $\mathrm{CM}$ distance between PAI-1 and SMB-IDD (27.5 $\AA$ ) is larger than the predicted value $(18 \pm 1 \AA)$. 


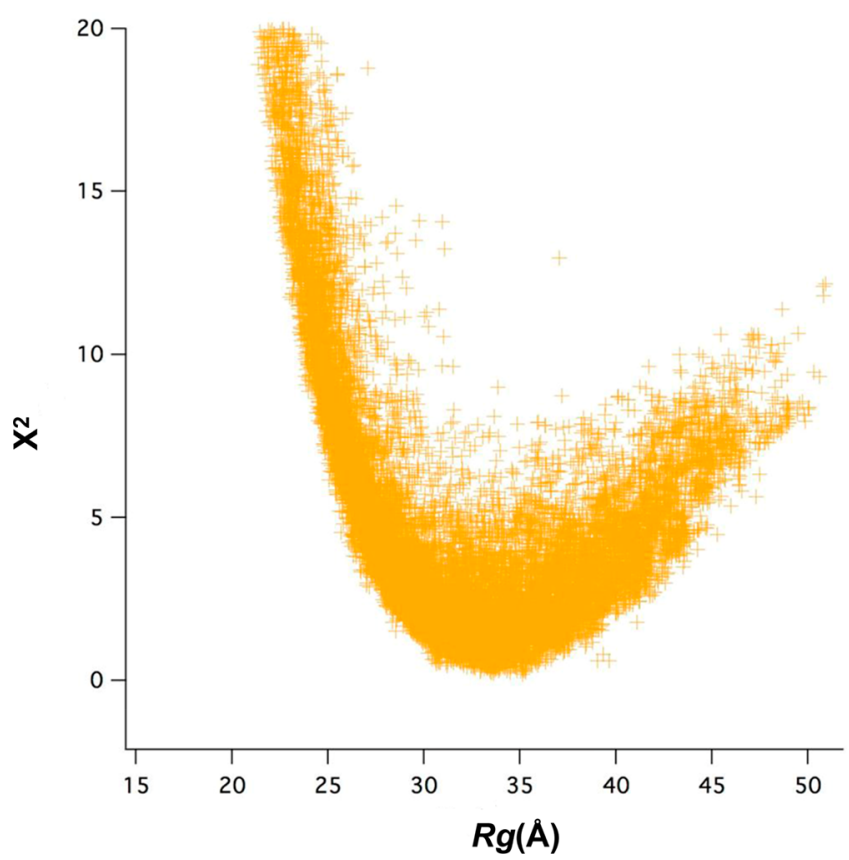

Figure 7. $\chi^{2}$ vs $R_{\mathrm{g}}$ plot for SANS on the unbound SMB-IDD. The free SMB-IDD starting structure, shown in Figure 5, was used to generate a large pool as described in Materials and Methods. As one can see in the $\chi^{2}$ vs $R_{\mathrm{g}}$ plot, scaled only to show $\chi^{2}$ values of $<20$, the structural pool generated from the SMB-IDD starting structure contains calculated structures that fit reasonably well to the SANS data for free SMB-IDD in solution.
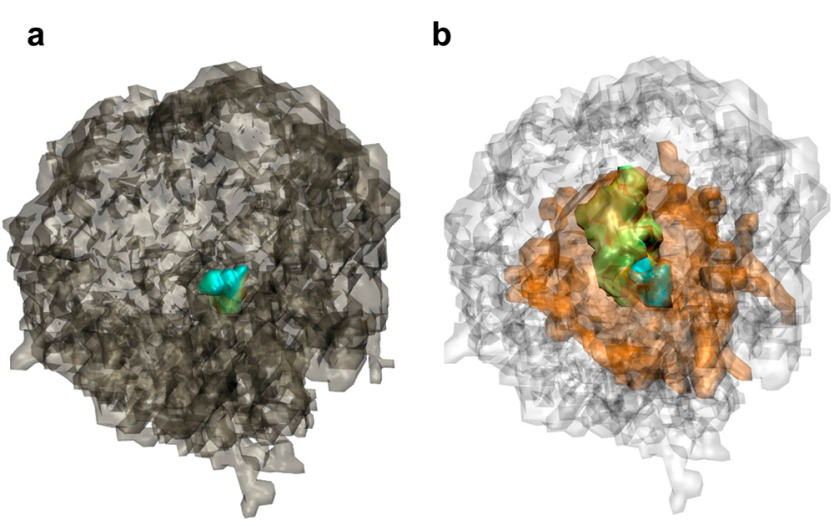

Figure 8. Density plots for unbound and bound SMB-IDD. (a) Unbound SMB-IDD. The SMB region is colored cyan. The gray cloud corresponds to unbound IDD structures with a $\chi^{2}$ value of $<1.5$. (b) PAI-1:SMB-IDD complex. PAI-1 bound to the SMB domain is shown at the center with PAI-1 colored green and the SMB domain colored cyan. The amber-colored cloud displays the IDD region for the 196 best fit structures for the $85 \% \mathrm{D}_{2} \mathrm{O}$ data, with a $\chi^{2}$ value of $<4$. For comparison, the SMB region for the PAI-1:SMB-IDD complex is superimposed on the SMB region for SBM-IDD alone in solution, and the same conformational space for the unbound IDD is colored lighter gray. The data demonstrate that, in the best fit structures, the IDD occupies a smaller range of three-dimensional space surrounding PAI-1:SMB at the core of the PAI-1:SMB-IDD complex relative to the space sampled by the structural ensemble for unbound SMB-IDD.

Although individual structures are an imprecise method for visualizing a complex with a large amount of flexibility, they aid in understanding complex formation. Therefore, we have chosen to highlight several of the 30 best fit structures at all three contrasts as representative conformational states in

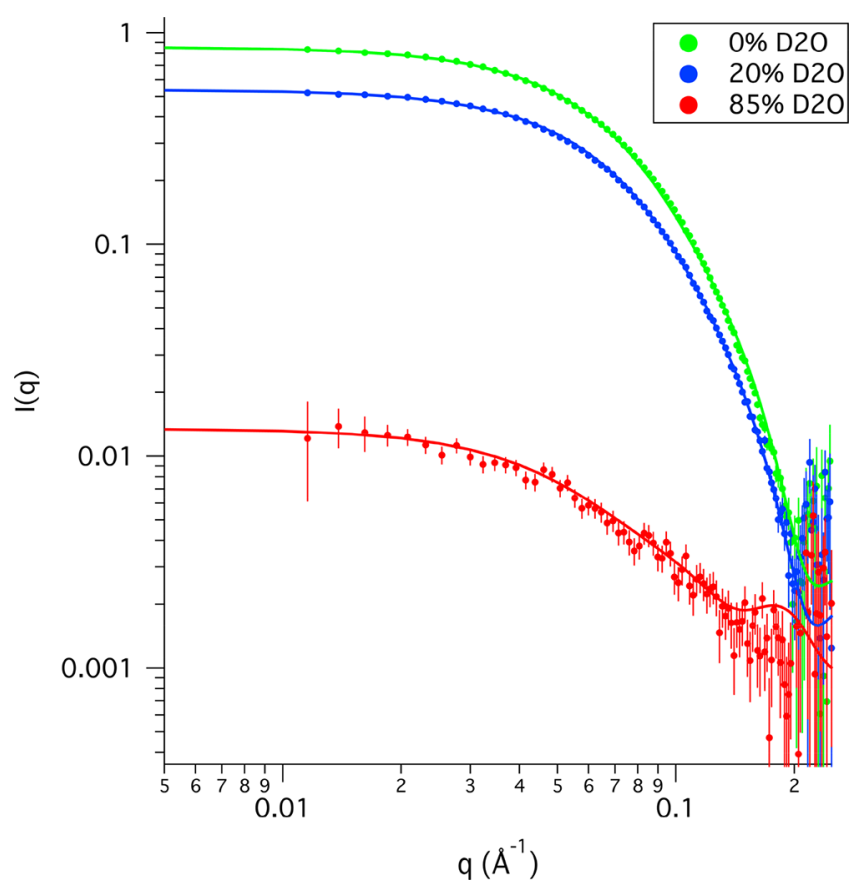

Figure 9. SANS data curves. Experimental SANS data are shown as dots for the three contrast conditions used for the structure modeling. The solid lines are the calculated SANS curves for the best fit structure to the $85 \% \mathrm{D}_{2} \mathrm{O}$ data, with $\chi^{2}=1.29$, plotted for all three contrasts. Error bars represent standard errors of the mean.

Figure 11. The best fit structure (number 16) is located on the bottom left. The 30 structures from the best fit data set (Table S3) share some common features. First, the region of the IDD that is proximal in sequence to the SMB domain is more restricted in conformational space compared to the more distal regions of the IDD. The proximal IDD regions consistently make a close approach to PAI-1, with a variety of conformations represented in the examples shown in Figure 11. There appears to be much more conformational freedom available to the distal IDD regions, which have variable to little contact with PAI-1. Thus, the inherent flexibility of the IDD is apparent from the SANS analysis. However, with full-length vitronectin, the IDD is anchored on the C-terminal end by the central $\beta$-propeller domain and will have more limited conformational freedom.

\section{DISCUSSION}

Vitronectin Contains an Intrinsically Disordered Domain. We have a long-standing interest in understanding the folding of vitronectin into individual domains and the assignments of functions within domains. A domain organization for vitronectin was originally proposed solely on the basis of sequence alignment ${ }^{24,101}$ and was supported by computational queries using threading approaches, which yielded a model with three domains in human vitronectin. ${ }^{1}$ Even in these early studies, the inability to provide a structural model for the $\sim 80$ amino acids that connected the N-terminal and central domains prohibited the assembly of a full-length model for vitronectin. Consequently, when intact vitronectin was analyzed using small-angle X-ray scattering (SAXS) to characterize the overall folding envelope, this $\sim 80$-amino acid linker sequence was reanalyzed using bioinformatics methods, with the ultimate conclusion that the region was unstructured. The unstructured character of this linker region agrees with a 

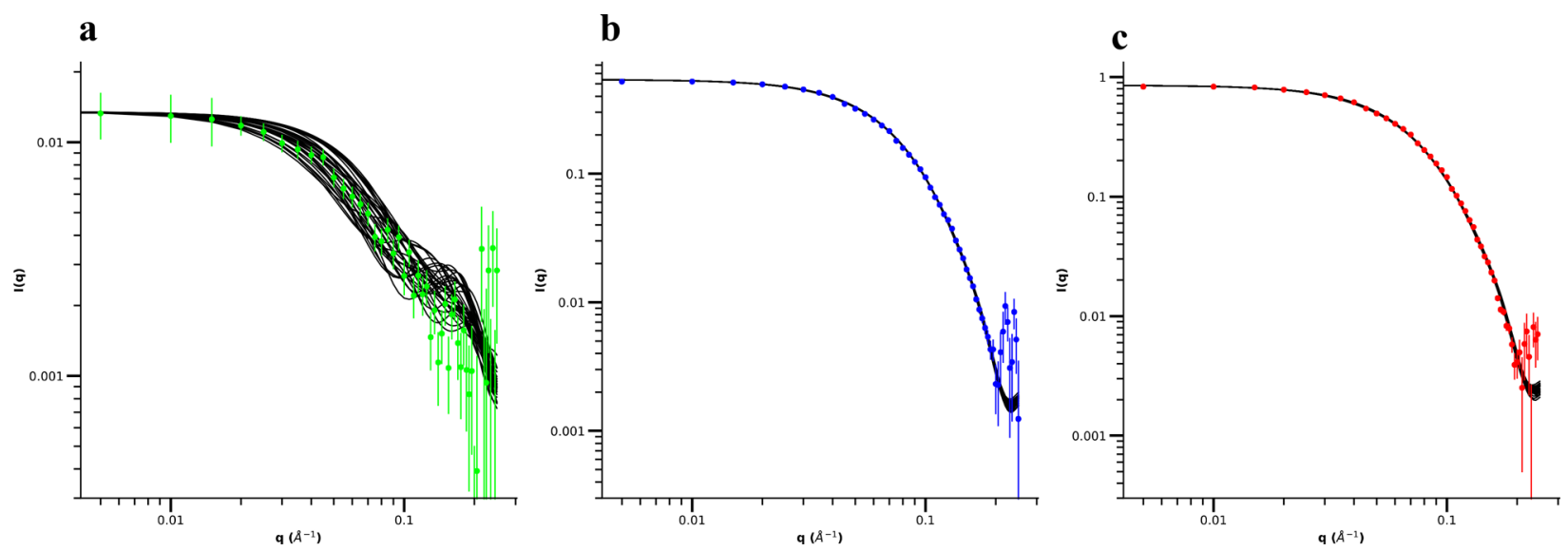

Figure 10. Calculated SANS curves for the best fit structures at all contrasts. Calculated SANS curves from the 30 structures that are in the best fit $\chi^{2}$ ensembles at all contrasts: (a) $85 \% \mathrm{D}_{2} \mathrm{O}$, best fit ensemble defined as $\chi^{2}<4$; (b) $20 \% \mathrm{D}_{2} \mathrm{O}$, best fit ensemble defined as $\chi^{2}<15$; and (c) $0 \%$ $\mathrm{D}_{2} \mathrm{O}$, best fit ensemble defined as $\chi^{2}<20$. Error bars represent standard errors of the mean.
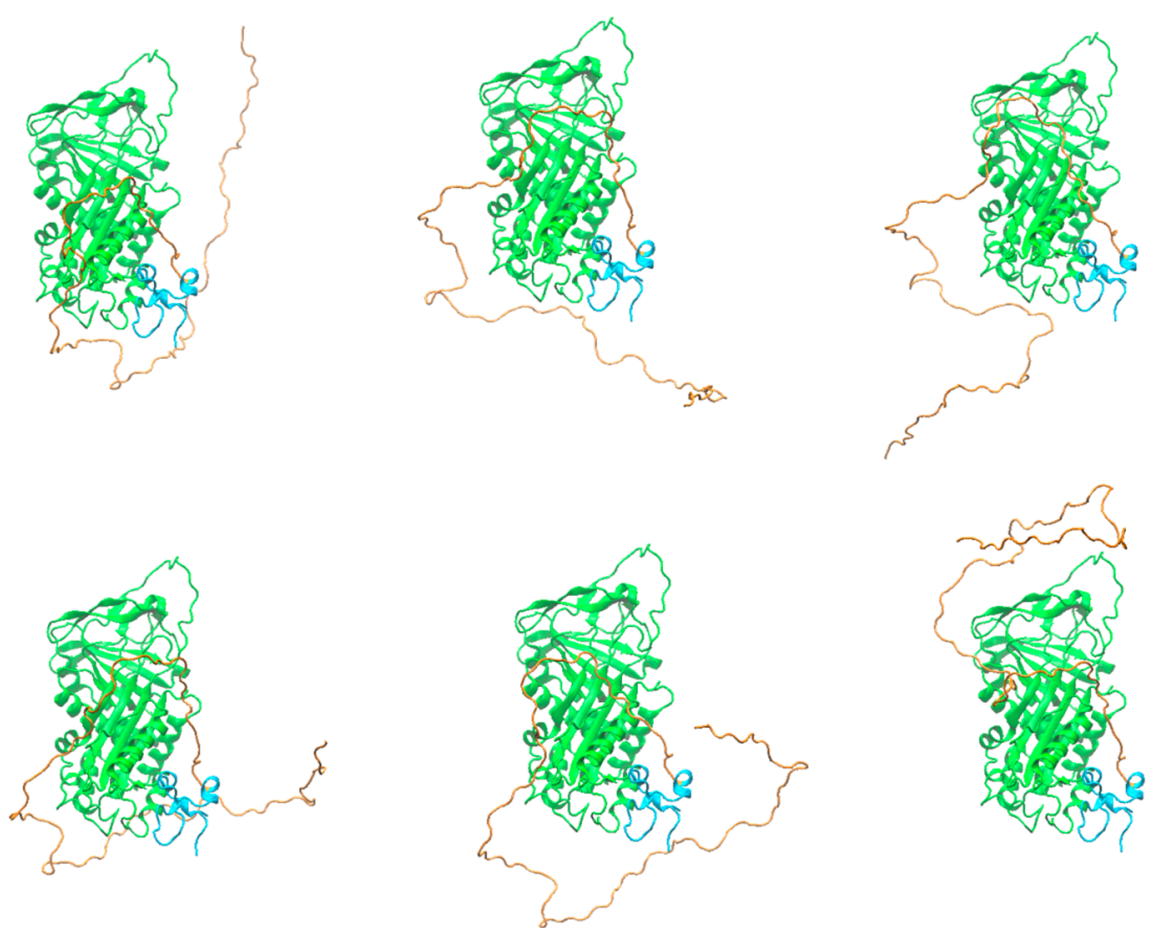

Figure 11. Representative structures for the PAI-1:SMB-IDD complex from the best fit ensemble at all contrasts. Six structures from the total 30 best fit structures at all contracts are shown, corresponding to structure 7 (top left), structure 8 (top middle), structure 13 (top right), structure 16 (bottom left), structure 20 (bottom middle), and structure 26 (bottom right) from Table S3. The overall best fit structure is structure 16 , with a $\chi^{2}$ value of $1.29\left(85 \% \mathrm{D}_{2} \mathrm{O}\right)$. PAI-1 is colored green, the SMB cyan, and the IDD amber. The structures consistently show that the IDD region proximal to the SMB makes close approaches to PAI-1, with the potential to form contacts with $\alpha$-helices D and E in PAI-1. It is clear from these representative structures that there is a large variation in the orientation of the distal end of the IDD.

variety of studies that have demonstrated its high susceptibility to proteolysis. ${ }^{1}$ The SAXS experiments defined a structural envelope characterized by a bilobed structure with an average $R_{\mathrm{g}}$ of $30.3 \pm 0.6 \AA$ and a maximum length of $110 \AA^{26}$ Interestingly, the narrowed region between the two lobes appeared to be occupied by the linker sequence, which was simply modeled as a cylinder that could be occupied by an indeterminant, flexible structure.

When the bilobed structure of vitronectin was described, we noted the benefit that a relatively disordered connecting region could provide flexibility between the two globular lobes. A disorder-to-order transition in this linker region was postulated to modulate specificity and/or affinity in ligand binding. Over the years, interest has developed in intrinsically disordered proteins, which are prone to such disorder-to-order transitions, typically in a coupled binding-folding event. Studies of intrinsically disordered proteins are becoming more functionally relevant. We are now aware that intrinsically disordered proteins display noteworthy functions in neural development, synaptic transmission, and cell cycle regulation, ${ }^{102,103}$ and they are embedded within many scaffolding proteins and also have roles in signaling sequences. ${ }^{35,36,104-106}$ Intrinsic disorder is emerging as a key feature in the versatility of living systems, whereby simultaneous promiscuity and specificity in binding 
interactions are manifested. ${ }^{107}$ While novel functions can be attributed to many IDD regions, the role of disordered regions is not always apparent.

The linker region in vitronectin has hallmark features of intrinsically disordered protein sequences. In particular, it harbors a significant number of acidic residues (Asp and Glu), has a net negative charge, and exhibits charge repulsion interactions. ${ }^{28}$ The hypotheses tested in this work are whether, in fact, this region can be classified as an IDD region and whether it undergoes a transition to adopt structure as we originally predicted. To query the classification of the interdomain linker in $\mathrm{VN}$ as an IDD capable of adopting structure, we used bioinformatic tools and experimental tests. The use of multiple computational tools designed for predicting the intrinsic disorder predisposition of a protein on a per-residue basis revealed that IDD is expected to be highly disordered. The inherent versatility of IDD regions is attributed to their ability to adopt diverse structures when they interact with different binding partners. The disordered regions that are prone to adopt such structures are denoted as Molecular Recognition Features (MoRFs), ${ }^{12}$ and MoRF prediction algorithms have pointed to segments of the $\mathrm{VN}$ IDD that can potentially gain structure upon ligand binding.

Along with these predictions, we were able to demonstrate experimentally that the linker region is disordered and undergoes a disorder-to-order transition. Both $\mathrm{CD}$ and SANS experiments confirm that the IDD, being highly disordered in aqueous solution, adopts structure and becomes more compact when exposed to osmotic pressure. Therefore, the computational modeling and experimental data support the classification of the linker region between the first and third domains of $\mathrm{VN}$ as an IDD region that gains structure under given circumstances. Within the three-dimensional model for VN generated by Lynn et al. from SAXS, NMR, and threading predictions, ${ }^{26}$ the narrowest point in the bilobed model where the IDD was mapped measures $\sim 35 \AA$. For comparison, the SANS data indicate that the free SMB-IDD exhibits an average $R_{\mathrm{g}}$ of $\sim 31 \AA$ with a maximum linear dimension of $\sim 78 \AA$. This classification of the linker as an IDD provided a valuable insight into this understudied domain of $\mathrm{VN}$ and prompted further studies to test whether binding of the PAI-1 ligand leads to a similar adoption of structure.

The Intrinsically Disordered Domain Houses a Site for PAI-1 Binding. Localization and further characterization of mutual interaction sites for PAI-1 and VN are important features of this study. While the identity of PAI-1-binding sites on vitronectin had been debated in early work, the primary high-affinity site for binding had been isolated within the SMB domain of vitronectin and the identity of other "secondary" sites for interaction remained uncertain but mostly ignored. However, upon our identification of additional vitronectinbinding sites on PAI-1, which were located outside of the flexible joint region, ${ }^{41}$ it became clear that a complementary binding site outside of the well-characterized SMB domain of vitronectin must be present. We judged the flexible linker, now known to be an IDD region, as a probable location for such a binding site because of its complementary charge character and inherent flexibility. In a series of SANS experiments to test for structural changes in the SMB-IDD, it was observed that PAI-1 binding led to structural reorganization of the IDD into a more compact structure. This result localizes an additional binding site for PAI-1 to the linker region in vitronectin.
Computational approaches with the PAI-1:SMB-IDD complex were used to provide models that conform to the SANS data measured with a series of solvents that differed in $\mathrm{D}_{2} \mathrm{O}$ levels in the contrast variation studies. The conformational space that is occupied in the density plots comparing both the free and bound SMB-IDD (Figure 8) illustrates a coupled folding-binding event with PAI-1 that leads to a more compact IDD region. Among the ensemble of 30 best fit structures for the PAI-1:SMB-IDD complex (Figure 11), the examples pictured illustrate close contacts that occur with PAI1 via the IDD region. In these representative structures from the best fit set (Table S3), a segment of the IDD sequence that is proximal to the SMB domain adopts an orientation that would allow contacts with helices D and E of PAI-1. This is noteworthy because the basic residues that comprise the more extensive binding site for vitronectin outside of the SMB domain are located on these particular $\alpha$-helices. ${ }^{41}$ While it may be tempting to investigate these models to identify potential binding residues, the resolution of SANS data does not warrant this detailed query.

A Comprehensive Model of PAI-1:VN Binding. Recognizing the noteworthy effects that PAI-1 and vitronectin confer on each other regarding function, assembly of complexes, and tissue localization and specificity, we have pursued a variety of approaches to define the binding interfaces and to characterize conformational changes and association to higher-order structures. For example, vitronectin localizes PAI1 to fibrin clots, ${ }^{43}$ promotes accretion of PAI-1 in the ECM, ${ }^{108}$ and broadens the spectrum of PAI-1 inhibition to target other serine proteases involved in coagulation. ${ }^{109,110}$ Also, PAI- 1 and vitronectin are localized together in the ECM in a variety of pathologies, including inflammation, tumor development, angiogenesis, and necrosis. ${ }^{111-113}$ Vitronectin found in the ECM adopts an oligomeric form ${ }^{114}$ and can engage a variety of cell surface receptors. Through analyses of the effects of PAI-1 on vitronectin over a $>24 \mathrm{~h}$ time course, we established that PAI-1 promotes the assembly of higher-order oligomers that exhibit enhanced interactions with integrins and ECM components. ${ }^{115}$ We also used analytical ultracentrifugation to demonstrate that PAI-1 and vitronectin interact in a 2:1 stoichiometry and assemble to higher-order multimers via a discrete $4: 2$ complex. $^{100}$

A model for association of these PAI-1:vitronectin complexes suggested multiple binding sites and conformational changes that promote higher-order assemblies. ${ }^{100}$ Stoppedflow kinetics established that full-length vitronectin and PAI-1 interact in two phases, whereas the SMB domain alone exhibits only one phase in binding. ${ }^{37}$ Conformational changes were detected in both proteins upon binding, and these structural changes occurred on a slower time frame compared to the initial binding phases. ${ }^{37}$ Such conformational changes are proposed to be essential for association of the altered, oligomeric form of vitronectin. Furthermore, the conformational change induced by the binding of full-length vitronectin to PAI-1 differed from that induced by the SMB domain alone. Also, the rapid reaction kinetics for attack of target proteases differed comparing free PAI-1 with complexes containing vitronectin or the SMB domain. ${ }^{37}$ These data were some of the first to demonstrate that a binding site for PAI-1 existed outside of the SMB domain and offered direct evidence of a folding-binding event leading to an altered structure upon binding of PAI-1 to vitronectin. 
Compelling support for a second PAI-1-binding site on vitronectin came from our studies of a form of vitronectin $(\mathrm{r} \Delta \mathrm{sBVN})$ that had the somatomedin $\mathrm{B}$ domain deleted yet retained PAI-1 binding. ${ }^{40}$ The $\mathrm{r} \Delta \mathrm{sBVN}$ protein partially inhibited binding of PAI-1 to full-length vitronectin and exhibited a $K_{\mathrm{d}}$ of $30 \mathrm{nM}$ (much weaker than binding of intact, full-length vitronectin containing the somatomedin $\mathrm{B}$ domain, which is $\sim 0.3 \mathrm{nM}^{116-120}$ ). The $\mathrm{r} \Delta \mathrm{sBVN}$ protein was used to map the complementary site on PAI-1 to a region outside of the somatomedin $\mathrm{B}$ binding area and localize it to the distal ends of $\alpha$-helices D and E. ${ }^{41}$ For the study presented here, another form of vitronectin with a deleted sequence, which contains the SMB domain and the $\sim 80$-amino acid linker but lacks the rest of the $\mathrm{C}$-terminus, was studied. This linker region is present in both the SMB-IDD and the $r \Delta s B V N$ studied previously. Thus, the SMB-IDD was generated to determine whether the IDD accounts for the PAI-1-binding epitope in $\mathrm{r} \triangle \mathrm{sBVN}$. The data from SANS are consistent with this idea, with the best fit structures for the SMB-IDD observed to be more compact upon binding. Thus, the data suggest that the SMB-IDD exhibits the expected coupled folding-binding behavior.

The study with the $r \Delta \mathrm{sBVN}$ form of $\mathrm{VN}$ also demonstrated a salt dependence in the assembly of the higher-order PAI1 :vitronectin multimers, with higher salt concentrations disfavoring assembly to the higher-order species. ${ }^{40}$ In contrast, ionic strength does not strictly disrupt the binding of PAI-1 to intact vitronectin, ${ }^{115}$ presumably because the contacts identified between amino acids in the flexible joint region and residues in the SMB domain are not sensitive to salt concentrations. The structural models for the PAI-1:SMB-IDD complex generated from the SANS analysis demonstrate a close approach of regions of the IDD with PAI-1 beyond the SMB-binding region. As noted above, the more extensive vitronectin-binding site recently identified outside the flexible joint region on PAI-1 contains several basic amino acids within helices D and $E,{ }^{41}$ and the IDD hosts a number of complementary acidic residues within regions that make a close approach in the structural models from this SANS work (Figure 11). The results from this SANS analysis have been expanded with a complementary protein engineering and kinetics approach to study PAI-1:vitronectin binding. ${ }^{46}$ This parallel study utilized a vitronectin fragment containing a truncated IDD to demonstrate a biphasic binding event with the SMB-IDD, narrowing down the binding site within the IDD to a 24-amino acid region and utilizing molecular dynamics to identify four charge:charge interactions within the binding site. ${ }^{46}$ Thus, the binding between charged residues in the IDD and amino acids within the more extensive binding epitope on PAI- $1^{41}$ should be disrupted by salt and prevent the coupled folding-binding event observed in this work.

Over many years, we have worked to address the hypothesis that stepwise binding of PAI-1, in a concentration-dependent pathway, is required to form higher-order PAI-1:vitronectin complexes. This SANS study contributes to a unifying model that is consistent with the large body of cumulative work on the binding of vitronectin to PAI-1 that has the following steps in a time sequence: (1) rapid association of PAI-1 with vitronectin in a concentration-dependent, biphasic fashion, (2) conformational changes in both PAI-1 and vitronectin, (3) assembly of higher-order complexes with multimers of vitronectin that persist in an altered conformation, and (4) dissociation of latent PAI-1 from the complexes on a slow time scale that mirrors the rate of decay of PAI- 1 from the active to latent form. Evidence from our concurrent study demonstrates that the PAI-1-binding site housed in the IDD contributes to the initial biphasic binding. ${ }^{46}$ The structural changes in this region are proposed to transduce structural changes throughout $\mathrm{VN}$, leading to the altered multimeric structure that is most effective in binding to ECM components and cell surface receptors. The characterization of an IDD in $\mathrm{VN}$ provides insight into the adaptability that $\mathrm{VN}$ may exhibit in binding ligands and in biological functions.

\section{ASSOCIATED CONTENT}

S Supporting Information

The Supporting Information is available free of charge at https://pubs.acs.org/doi/10.1021/acs.biochem.9b00605.

Figures S1-S5 and Tables S1-S3 (PDF)

\section{Accession Codes}

PAI-1, UniProtKB P05121 (PAI1 HUMAN); vitronectin, UniProtKB P04004 (VTNC_HUMĀN).

\section{AUTHOR INFORMATION}

\section{Corresponding Author}

*E-mail: cbpeterson@lsu.edu. Telephone: (225) 578-4202.

ORCID

Christopher B. Stanley: 0000-0002-4226-7710

Vladimir N. Uversky: 0000-0002-4037-5857

Cynthia B. Peterson: 0000-0002-9220-753X

Funding

A portion of this research at ORNL's High Flux Isotope Reactor and Spallation Neutron Source was sponsored by the Scientific User Facilities Division, Office of Basic Energy Sciences, U.S. Department of Energy (DOE). Research in the Biodeuteration lab at ORNL's Center for Structural Molecular Biology (CSMB) was supported by the Office of Biological and Environmental Research, using facilities supported by the DOE, managed by UT-Battelle, LLC, under Contract DEAC05-00OR22725. Support for S.K. was provided by the Center for High Resolution Neutron Scattering, a partnership between the National Institute of Standards and Technology and the National Science Foundation under Agreement DMR1508249 .

\section{Notes}

The authors declare no competing financial interest.

\section{ACKNOWLEDGMENTS}

This work benefitted from CCP-SAS software developed through a joint EPSRC (EP/K039121/1) and NSF (CHE1265821) grant.

\section{ABBREVIATIONS}

$\mathrm{CD}$, circular dichroism; CM, center of mass; ECM, extracellular matrix; EG, ethylene glycol; EOM, Ensemble Optimization Method; IDD, intrinsically disordered domain; IMAC, immobilized metal affinity chromatography; IPTG, isopropyl $\beta$-D-1-thiogalactopyranoside; MD, molecular dynamics; MoRF, Molecular Recognition Feature; PAI-1, plasminogen activator inhibitor-1; PEG, polyethylene glycol; RCL, reaction center loop; $R_{\mathrm{g}}$, radius of gyration; SANS, small-angle neutron scattering; SAXS, small-angle X-ray scattering; SLD, scattering-length density; SMB, somatomedin B; VN, vitronectin. 


\section{ADDITIONAL NOTE}

${ }^{a}$ Certain commercial equipment, instruments, materials, suppliers, and software are identified in this paper to foster understanding. Such identification does not imply recommendation or endorsement by the National Institute of Standards and Technology, nor does it imply that the materials or equipment identified is necessarily the best available for the purpose.

\section{REFERENCES}

(1) Xu, D., Baburaj, K., Peterson, C. B., and Xu, Y. (2001) Model for the three-dimensional structure of vitronectin: Predictions for the multi-domain protein from threading and docking. Proteins: Struct., Funct., Genet. 44, 312-320.

(2) Piovesan, D., Tabaro, F., Micetic, I., Necci, M., Quaglia, F., Oldfield, C. J., Aspromonte, M. C., Davey, N. E., Davidovic, R., Dosztanyi, Z., Elofsson, A., Gasparini, A., Hatos, A., Kajava, A. V., Kalmar, L., Leonardi, E., Lazar, T., Macedo-Ribeiro, S., MacossayCastillo, M., Meszaros, A., Minervini, G., Murvai, N., Pujols, J., Roche, D. B., Salladini, E., Schad, E., Schramm, A., Szabo, B., Tantos, A., Tonello, F., Tsirigos, K. D., Veljkovic, N., Ventura, S., Vranken, W., Warholm, P., Uversky, V. N., Dunker, A. K., Longhi, S., Tompa, P., and Tosatto, S. C. (2016) DisProt 7.0: a major update of the database of disordered proteins. Nucleic Acids Res. 45, D1123-D1124.

(3) Barnes, D., Wolfe, R., Serrero, G., McClure, D., and Sato, G. (1980) Effects of a serum spreading factor on growth and morphology of cells in serum-free medium. J. Supramol. Struct. 14, 47-63.

(4) Holmes, R. (1967) Preparation from human serum of an alphaone protein which induces immediate growth of unadapted cells in vitro. J. Cell Biol. 32, 297-308.

(5) Hayman, E. G., Pierschbacher, M. D., Ohgren, Y., and Ruoslahti, E. (1983) Serum spreading factor (vitronectin) is present at the cell surface and in tissues. Proc. Natl. Acad. Sci. U. S. A. 80, 4003-4007. (6) Barnes, D. W., Silnutzer, J., See, C., and Shaffer, M. (1983) Characterization of human serum spreading factor with monoclonal antibody. Proc. Natl. Acad. Sci. U. S. A. 80, 1362-1366.

(7) Kost, C., Benner, K., Stockmann, A., Linder, D., and Preissner, K. T. (1996) Limited plasmin proteolysis of vitronectin - Characterization of the adhesion protein as morpho-regulatory and Angiostatinbinding factor. Eur. J. Biochem. 236, 682-688.

(8) Podor, T. J., Campbell, S., Chindemi, P., Foulon, D. M., Farrell, D. H., Walton, P. D., Weitz, J. I., and Peterson, C. B. (2002) Incorporation of vitronectin into fibrin clots - Evidence for a binding interaction between vitronectin and gamma $\mathrm{A} /$ gamma ' fibrinogen. $J$. Biol. Chem. 277, 7520-7528.

(9) Defilippi, P., Truffa, G., Stefanuto, G., Altruda, F., Silengo, L., and Tarone, G. (1991) Tumor necrosis factor alpha and interferon gamma modulate the expression of the vitronectin receptor (integrin beta 3) in human endothelial cells. J. Biol. Chem. 266, 7638-7645.

(10) Schnapp, L. M., Hatch, N., Ramos, D. M., Klimanskaya, I. V., Sheppard, D., and Pytela, R. (1995) The human integrin alpha 8 beta 1 functions as a receptor for tenascin, fibronectin, and vitronectin. J. Biol. Chem. 270, 23196-23202.

(11) Smith, J. W., Vestal, D. J., Irwin, S. V., Burke, T. A., and Cheresh, D. A. (1990) Purification and functional characterization of integrin alpha v beta 5. An adhesion receptor for vitronectin. J. Biol. Chem. 265, 11008-11013.

(12) Disfani, F. M., Hsu, W.-L., Mizianty, M. J., Oldfield, C. J., Xue, B., Dunker, A. K., Uversky, V. N., and Kurgan, L. (2012) MoRFpred, a computational tool for sequence-based prediction and characterization of short disorder-to-order transitioning binding regions in proteins. Bioinformatics 28, I75-I83.

(13) Wei, Y., Waltz, D. A., Rao, N., Drummond, R. J., Rosenberg, S., and Chapman, H. A. (1994) Identification of the urokinase receptor as an adhesion receptor for vitronectin. J. Biol. Chem. 269, 3238032388 .
(14) Preissner, K. T., Wassmuth, R., and Mullerberghaus, G. (1985) Physicochemical characterization of human S-protein and its function in the blood coagulation system. Biochem. J. 231, 349-355.

(15) Declerck, P. J., Demol, M., Alessi, M. C., Baudner, S., Paques, E. P., Preissner, K. T., Mullerberghaus, G., and Collen, D. (1988) Purification and characterization of a plasminogen activator inhibitor 1 binding protein from human plasma. Identification as a multimeric form of S protein (vitronectin). J. Biol. Chem. 263, 15454-15461.

(16) Preissner, K. T. (1991) Structure and Biological Role of Vitronectin. Annu. Rev. Cell Biol. 7, 275-310.

(17) Felding-Habermann, B., and Cheresh, D. A. (1993) Vitronectin and its receptors. Curr. Opin. Cell Biol. 5, 864-868.

(18) Schvartz, I., Seger, D., and Shaltiel, S. (1999) Vitronectin. Int. J. Biochem. Cell Biol. 31, 539-544.

(19) Preissner, K. T., and Seiffert, D. (1998) Role of vitronectin and its receptors in haemostasis and vascular remodeling. Thromb. Res. 89, $1-21$.

(20) Tollefsen, D. M., Weigel, C. J., and Kabeer, M. H. (1990) The presence of methionine or threonine at position 381 in vitronectin is correlated with proteolytic cleavage at arginine 379. J. Biol. Chem. 265, 9778-9781.

(21) Gibson, A. D., and Peterson, C. B. (2001) Full-length and truncated forms of vitronectin provide insight into effects of proteolytic processing on function. Biochim. Biophys. Acta, Protein Struct. Mol. Enzymol. 1545, 289-304.

(22) Kamikubo, Y., De Guzman, R., Kroon, G., Curriden, S., Neels, J. G., Churchill, M. J., Dawson, P., Oldziej, S., Jagielska, A., Scheraga, H. A., Loskutoff, D. J., and Dyson, H. J. (2004) Disulfide bonding arrangements in active forms of the somatomedin B domain of human vitronectin. Biochemistry 43, 6519-6534.

(23) Mayasundari, A., Whittemore, N. A., Serpersu, E. H., and Peterson, C. B. (2004) The solution structure of the N-terminal domain of human vitronectin - Proximal sites that regulate fibrinolysis and cell migration. J. Biol. Chem. 279, 29359-29366.

(24) Suzuki, S., Pierschbacher, M. D., Hayman, E. G., Nguyen, K., Ohgren, Y., and Ruoslahti, E. (1984) Domain structure of vitronectin. Alignment of active sites. J. Biol. Chem. 259, 5307-5314.

(25) Zhou, A. (2007) Functional structure of the somatomedin B domain of vitronectin. Protein Sci. 16, 1502-1508.

(26) Lynn, G. W., Heller, W. T., Mayasundari, A., Minor, K. H., and Peterson, C. B. (2005) A model for the three-dimensional structure of human plasma vitronectin from small-angle scattering measurements. Biochemistry 44, 565-574.

(27) Preissner, K. T. (1991) Structure and Biological Role of Vitronectin. Annu. Rev. Cell Biol. 7, 275-310.

(28) Rani, P., Baruah, A., and Biswas, P. (2014) Does Lack of Secondary Structure Imply Intrinsic Disorder in Proteins? A Sequence Analysis. Biochim. Biophys. Acta, Proteins Proteomics 1844, 1827-1834.

(29) Dyson, H. J., and Wright, P. E. (2005) Intrinsically unstructured proteins and their functions. Nat. Rev. Mol. Cell Biol. 6, 197-208.

(30) Romero, P., Obradovic, Z., Li, X. H., Garner, E. C., Brown, C. J., and Dunker, A. K. (2001) Sequence complexity of disordered protein. Proteins: Struct., Funct., Genet. 42, 38-48.

(31) Vucetic, S., Brown, C. J., Dunker, A. K., and Obradovic, Z. (2003) Flavors of protein disorder. Proteins: Struct., Funct., Genet. 52, $573-584$.

(32) Dunker, A. K., Lawson, J. D., Brown, C. J., Williams, R. M., Romero, P., Oh, J. S., Oldfield, C. J., Campen, A. M., Ratliff, C. R., Hipps, K. W., Ausio, J., Nissen, M. S., Reeves, R., Kang, C. H., Kissinger, C. R., Bailey, R. W., Griswold, M. D., Chiu, M., Garner, E. C., and Obradovic, Z. (2001) Intrinsically disordered protein. J. Mol. Graphics Model. 19, 26-59.

(33) Wright, P. E., and Dyson, H. J. (1999) Intrinsically unstructured proteins: Re-assessing the protein structure-function paradigm. J. Mol. Biol. 293, 321-331.

(34) Uversky, V. N., Dave, V., Iakoucheva, L. M., Malaney, P., Metallo, S. J., Pathak, R. R., and Joerger, A. C. (2014) Pathological 
Unfoldomics of Uncontrolled Chaos: Intrinsically Disordered Proteins and Human Diseases. Chem. Rev. 114, 6844-6879.

(35) Uversky, V. N. (2015) The multifaceted roles of intrinsic disorder in protein complexes. FEBS Lett. 589, 2498-2506.

(36) Dunker, A. K., Oldfield, C. J., Meng, J., Romero, P., Yang, J. Y., Chen, J. W., Vacic, V., Obradovic, Z., and Uversky, V. N. (2008) The unfoldomics decade: an update on intrinsically disordered proteins. BMC Genomics 9 (Suppl. 2), S1.

(37) Blouse, G. E., Dupont, D. M., Schar, C. R., Jensen, J. K., Minor, K. H., Anagli, J. Y., Gardsvoll, H., Ploug, M., Peterson, C. B., and Andreasen, P. A. (2009) Interactions of Plasminogen Activator Inhibitor-1 with Vitronectin Involve an Extensive Binding Surface and Induce Mutual Conformational Rearrangements. Biochemistry 48, $1723-1735$.

(38) Zhou, A. W., Huntington, J. A., Pannu, N. S., Carrell, R. W., and Read, R. J. (2003) How vitronectin binds PAI-1 to modulate fibrinolysis and cell migration. Nat. Struct. Biol. 10, 541-544.

(39) Seiffert, D., Ciambrone, G., Wagner, N. V., Binder, B. R., and Loskutoff, D. J. (1994) The somatomedin B domain of vitronectin. Structural requirements for the binding and stabilization of active type 1 plasminogen activator inhibitor. J. Biol. Chem. 269, 2659-2666.

(40) Schar, C. R., Blouse, G. E., Minor, K. H., and Peterson, C. B. (2008) A deletion mutant of vitronectin lacking the somatomedin B domain exhibits residual plasminogen activator inhibitor-1-binding activity. J. Biol. Chem. 283, 10297-10309.

(41) Schar, C. R., Jensen, J. K., Christensen, A., Blouse, G. E., Andreasen, P. A., and Peterson, C. B. (2008) Characterization of a site on PAI-1 that binds to vitronectin outside of the somatomedin B domain. J. Biol. Chem. 283, 28487-28496.

(42) Schroeck, F., Arroyo de Prada, N., Sperl, S., Schmitt, M., and Magdolen, V. (2002) Interaction of plasminogen activator inhibitor type-1 (PAI-1) with vitronectin (Vn): Mapping the binding sites on PAI-1 and Vn. Biol. Chem. 383, 1143-1149.

(43) Podor, T. J., Shaughnessy, S. G., Blackburn, M. N., and Peterson, C. B. (2000) New insights into the size and stoichiometry of the plasminogen activator inhibitor type-1 vitronectin complex. J. Biol. Chem. 275, 25402-25410.

(44) Kost, C., Stuber, W., Ehrlich, H. J., Pannekoek, H., and Preissner, K. T. (1992) Mapping of binding sites for heparin, plasminogen activator inhibitor-1, and plasminogen to vitronectin's heparin-binding region reveals a novel vitronectin-dependent feedback mechanism for the control of plasmin formation. J. Biol. Chem. 267, 12098-12105.

(45) Mimuro, J., Muramatsu, S., Kurano, Y., Uchida, Y., Ikadai, H., Watanabe, S., and Sakata, Y. (1993) Identification of the plasminogen activator inhibitor-1 binding heptapeptide in vitronectin. Biochemistry 32, 2314-2320.

(46) Chu, Y., Bucci, J. C., and Peterson, C. B. (2019) Identification of a PAI-1 binding site within an intrinsically disordered region of vitronectin. Protein Sci., DOI: 10.1002/pro.3770.

(47) Costa, S., Almeida, A., Castro, A., and Domingues, L. (2014) Fusion tags for protein solubility, purification and immunogenicity in Escherichia coli: the novel Fh8 system. Front. Microbiol. 5, 63.

(48) Thompson, L. C., Goswami, S., Ginsberg, D. S., Day, D. E., Verhamme, I. M., and Peterson, C. B. (2011) Metals affect the structure and activity of human plasminogen activator inhibitor-1. I. Modulation of stability and protease inhibition. Protein Sci. 20, 353365.

(49) Jensen, J. K., Thompson, L. C., Bucci, J. C., Nissen, P., Gettins, P. G. W., Peterson, C. B., Andreasen, P. A., and Morth, J. P. (2011) Crystal structure of plasminogen activator inhibitor-1 in an active conformation with normal thermodynamic stability. J. Biol. Chem. 286, 29709-29717.

(50) Whitmore, L., and Wallace, B. A. (2004) DICHROWEB, an online server for protein secondary structure analyses from circular dichroism spectroscopic data. Nucleic Acids Res. 32, W668-W673.

(51) Whitmore, L., and Wallace, B. A. (2008) Protein secondary structure analyses from circular dichroism spectroscopy: Methods and reference databases. Biopolymers 89, 392-400.
(52) Heller, W. T., Urban, V. S., Lynn, G. W., Weiss, K. L., O'Neill, H. M., Pingali, S. V., Qian, S., Littrell, K. C., Melnichenko, Y. B., Buchanan, M. V., Selby, D. L., Wignall, G. D., Butler, P. D., and Myles, D. A. (2014) The Bio-SANS Instrument at the HIgh FLux Isotope Reactor of Oak Ridge National LAboratory. J. Appl. Crystallogr. 47, $1238-1246$.

(53) Zhao, J. K., Gao, C. Y., and Liu, D. (2010) The extended Qrange small-angel neutron scattering diffractometer at the SNS. $J$. Appl. Crystallogr. 43, 1068-1077.

(54) Arnold, O., Bilheux, J.C., Borreguero, J.M., Buts, A., Campbell, S.I., Chapon, L., Doucet, M., Draper, N., Ferraz Leal, R., Gigg, M.A., Lynch, V.E., Markvardsen, A., Mikkelson, D.J., Mikkelson, R.L., Miller, R., Palmen, K., Parker, P., Passos, G., Perring, T.G., Peterson, P.F., Ren, S., Reuter, M.A., Savici, A.T., Taylor, J.W., Taylor, R.J., Tolchenov, R., Zhou, W., and Zikovsky, J. (2014) Mantid - Data analysis and visulatization package for neutron scatterin and $\mathrm{u} S \mathrm{SR}$ experiments. Nucl. Instrum. Methods Phys. Res., Sect. A 764, 156-166.

(55) Wignall, G. E., and Bates, F. S. (1987) Absolute calibration of small-angle neutron scattering data. J. Appl. Crystallogr. 20, 28-40.

(56) Guinier, A., and Fournet, G. (1955) Small-angle scattering of $X$ rays, John Wiley \& Sons, Inc., New York.

(57) Whitten, E., Cai, S., and Trewhella, J. (2008) MULCh: modules for the analysis of small-angle neutron contrast variation data from biomolecular assemblies. J. Appl. Crystallogr. 41, 222-226.

(58) Sarachan, K. L., Curtis, J. E., and Krueger, S. (2013) Smallangle scattering contrast calculator for protein and nucleic acid complexes in solution. J. Appl. Crystallogr. 46, 1889-1893.

(59) Ibel, K., and Stuhrmann, H. B. (1975) Comparison of Neutron and X-ray Scattering of Dilute Myoglobin Solutions. J. Mol. Biol. 93, $255-265$.

(60) Moore, P. B. (1982) Small-angle scattering techniques for the study of biological macromolecules and macromolecular aggregates. In Methods of Experimental Physics (Lecar, G. E. H., Ed.) pp 337-390, Academic Press, New York.

(61) Engelman, D., and Moore, P. B. (1975) Determination of Quaternary Structure by Small-Angle Neutron Scattering. Annu. Rev. Biophys. Bioeng. 4, 219-241.

(62) Bernado, P., Mylonas, E., Petoukhov, M. V., Blackledge, M., and Svergun, D. I. (2007) Structural characterization of flexible proteins using small-angle X-ray scattering. J. Am. Chem. Soc. 129, 5656-5664.

(63) Petoukhov, M. V., Franke, D., Shkumatov, A. V., Tria, G., Kikhney, A. G., Gajda, M., Gorba, C., Mertens, H. D. T., Konarev, P. V., and Svergun, D. I. (2012) New developments in the ATSAS program package for small-angle scattering data analysis. J. Appl. Crystallogr. 45, 342-350.

(64) Bernado, P., Mylonas, E., Petoukhov, M. V., Blackledge, M., and Svergun, D. I. (2007) Structural characterization of flexible proteins using small-angle X-ray scattering. J. Am. Chem. Soc. 129, 5656-5664.

(65) Svergun, D. I., Richard, S., Koch, M. H. J., Sayers, Z., Kuprin, S., and Zaccai, G. (1998) Protein hydration in solution: Experimental observation by $\mathrm{x}$-ray and neutron scattering. Proc. Natl. Acad. Sci. U. S. A. 95, 2267-2272.

(66) Tria, G., Mertens, H. D. T., Kachala, M., and Svergun, D. I. (2015) Advanced ensemble modelling of flexible macromolecules using X-ray solution scattering. IUCrJ 2, 207-217.

(67) Svergun, D., Barberato, C., and Koch, M. H. J. (1995) CRYSOL - a Program to Evaluate X-ray Solution Scattering of Biological Macromolecules from Atomic Coordinates. J. Appl. Crystallogr. 28, 768-773.

(68) Curtis, J. E., Raghunandan, S., Nanda, H., and Krueger, S. (2012) SASSIE: A program to study intrinsically disordered biological molecules and macromolecular ensembles using experimental scattering restraints. Comput. Phys. Commun. 183, 382-389.

(69) Phillips, J. C., Braun, R., Wang, W., Gumbart, J., Tajkhorshid, E., Villa, E., Chipot, C., Skeel, R. D., Kale, L., and Schulten, K. (2005) Scalable molecular dynamics with NAMD. J. Comput. Chem. 26, $1781-1802$. 
(70) Chen, J., Im, W., and Brooks, C. L. (2005) Application of torsion angle molecular dynamics for efficient sampling of protein conformations. J. Comput. Chem. 26, 1565-1578.

(71) Kjaergaard, M., Gardsvoll, H., Hirschberg, D., Nielbo, S., Mayasundari, A., Peterson, C. B., Jansson, A., Jorgensen, T. J. D., Poulsen, F. M., and Ploug, M. (2007) Solution structure of recombinant somatomedin B domain from vitronectin produced in Pichia pastoris. Protein Sci. 16, 1934-1945.

(72) Jones, D. T. (1999) Protein secondary structure prediction based on position-specific scoring matrices. J. Mol. Biol. 292, 195202.

(73) Kelley, L. A., and Sternberg, M. J. E. (2009) Protein structure prediction on the Web: a case study using the Phyre server. Nat. Protoc. 4, 363-371.

(74) Kelley, L. A., Mezulis, S., Yates, C. M., Wass, M. N., and Sternberg, M. J. E. (2015) The Phyre2 web portal for protein modeling, prediction and analysis. Nat. Protoc. 10, 845-858.

(75) Fasman, G. D. (1985) Prediction of protein structure from sequence. Nature 316, 22-22.

(76) Garnier, J., Osguthorpe, D. J., and Robson, B. (1978) Analysis of the accuracy and implications of simple methods for predicting the secondary structure of globular proteins. J. Mol. Biol. 120, 97-120.

(77) Buchan, D. W. A., Minneci, F., Nugent, T. C. O., Bryson, K., and Jones, D. T. (2013) Scalable web services for the PSIPRED Protein Analysis Workbench. Nucleic Acids Res. 41, W349-W357.

(78) Humphrey, W., Dalke, A., and Schulten, K. (1996) VMD: Visual molecular dynamics. J. Mol. Graphics 14, 33-38.

(79) MacKerell, A. D., Bashford, D., Bellott, M., Dunbrack, R. L., Evanseck, J. D., Field, M. J., Fischer, S., Gao, J., Guo, H., Ha, S., Joseph-McCarthy, D., Kuchnir, L., Kuczera, K., Lau, F. T., Mattos, C., Michnick, S., Ngo, T., Nguyen, D. T., Prodhom, B., Reiher, W. E., Roux, B., Schlenkrich, M., Smith, J. C., Stote, R., Straub, J., Watanabe, M., Wiorkiewicz-Kuczera, J., Yin, D., and Karplus, M. (1998) All-atom empirical potential for molecular modeling and dynamics studies of proteins. J. Phys. Chem. B 102, 3586-3616.

(80) Watson, M. C., and Curtis, J. E. (2013) Rapid and accurate calculation of small-angle scattering profiles using the golden ratio. $\mathrm{J}$. Appl. Crystallogr. 46, 1171-1177.

(81) Romero, P., Obradovic, Z., Li, X., Garner, E. C., Brown, C. J., and Dunker, A. K. (2001) Sequence complexity of disordered protein. Proteins: Struct., Funct., Genet. 42, 38-48.

(82) Peng, K., Radivojac, P., Vucetic, S., Dunker, A. K., and Obradovic, Z. (2006) Length-dependent prediction of protein intrinsic disorder. BMC Bioinf. 7, 208.

(83) Obradovic, Z., Peng, K., Vucetic, S., Radivojac, P., Brown, C. J., and Dunker, A. K. (2003) Predicting intrinsic disorder from amino acid sequence. Proteins: Struct., Funct., Genet. 53, 566-572.

(84) Xue, B., Dunbrack, R. L., Williams, R. W., Dunker, A. K., and Uversky, V. N. (2010) PONDR-FIT: A meta-predictor of intrinsically disordered amino acids. Biochim. Biophys. Acta, Proteins Proteomics 1804, 996-1010.

(85) Dosztanyi, Z., Csizmok, V., Tompa, P., and Simon, I. (2005) IUPred: web server for the prediction of intrinsically unstructured regions of proteins based on estimated energy content. Bioinformatics $21,3433-3434$.

(86) Dosztanyi, Z., Csizmok, V., Tompa, P., and Simon, I. (2005) The pairwise energy content estimated from amino acid composition discriminates between folded and intrinsically unstructured proteins. J. Mol. Biol. 347, 827-839.

(87) Permyakov, S. E., Vologzhannikova, A. A., Khorn, P. A., Shevelyova, M. P., Kazakov, A. S., Emelyanenko, V. I., Denesyuk, A. I., Denessiouk, K., Uversky, V. N., and Permyakov, E. A. (2018) Comprehensive analysis of the roles of 'black' and 'gray' clusters in structure and function of rat beta-parvalbumin. Cell Calcium 75, 6478.

(88) Deryusheva, E. I., Denesyuk, A. I., Denessiouk, K., Uversky, V. N., Permyakov, S. E., and Permyakov, E. A. (2018) On the relationship between the conserved 'black' and 'gray' structural clusters and intrinsic disorder in parvalbumins. Int. J. Biol. Macromol. $120,1055-1062$

(89) Walsh, I., Giollo, M., Di Domenico, T., Ferrari, C. Zimmermann, O., and Tosatto, S. C. (2015) Comprehensive largescale assessment of intrinsic protein disorder. Bioinformatics 31, 201208

(90) Fan, X., and Kurgan, L. (2014) Accurate prediction of disorder in protein chains with a comprehensive and empirically designed consensus. J. Biomol. Struct. Dyn. 32, 448-464.

(91) Peng, Z., and Kurgan, L. (2012) On the complementarity of the consensus-based disorder prediction. Pacific Symposium on Biocomputing. Pacific Symposium on Biocomputing, 176-187.

(92) van der Lee, R., Buljan, M., Lang, B., Weatheritt, R. J., Daughdrill, G. W., Dunker, A. K., Fuxreiter, M., Gough, J., Gsponer, J., Jones, D. T., Kim, P. M., Kriwacki, R. W., Oldfield, C. J., Pappu, R. V., Tompa, P., Uversky, V. N., Wright, P. E., and Babu, M. M. (2014) Classification of intrinsically disordered regions and proteins. Chem. Rev. 114, 6589-6631.

(93) Parsegian, V. A., Rand, R. P., and Rau, D. C. (1995) Macromolecules and water: Probing with osmotic stress. Methods Enzymol. 259, 43-94.

(94) Stanley, C., Krueger, S., Parsegian, V. A., and Rau, D. C. (2008) Protein structure and hydration probed by SANS and osmotic stress. Biophys. J. 94, 2777-2789.

(95) Parsegian, V. A., Rand, R. P., and Rau, D. C. (2000) Osmotic stress, crowding, preferential hydration, and binding: A comparison of perspectives. Proc. Natl. Acad. Sci. U. S. A. 97, 3987-3992.

(96) Mansouri, A. L., Grese, L. N., Rowe, E. L., Pino, J. C., Chennubhotla, S. C., Ramanathan, A., O’Neill, H. M., Berthelier, V., and Stanley, C. B. (2016) Folding propensity of intrinsically disordered proteins by osmotic stress. Mol. BioSyst. 12, 3695-3701.

(97) Uversky, V. N. (2013) Unusual biophysics of intrinsically disordered proteins. Biochim. Biophys. Acta, Proteins Proteomics 1834, 932-951.

(98) Kuznetsova, I. M., Turoverov, K. K., and Uversky, V. N. (2014) What macromolecular crowding can do to a protein. Int. J. Mol. Sci. $15,23090-23140$.

(99) Politi, R., and Harries, D. (2010) Enthalpically driven peptide stabilization by protective osmolytes. Chem. Commun. (Cambridge, $U$. K.) 46, 6449-6451.

(100) Minor, K. H., Schar, C. R., Blouse, G. E., Shore, J. D., Lawrence, D. A., Schuck, P., and Peterson, C. B. (2005) A mechanism for assembly of complexes of vitronectin and plasminogen activator inhibitor-1 from sedimentation velocity analysis. J. Biol. Chem. 280, 28711-28720.

(101) Hunt, L. T., Barker, W. C., and Chen, H. R. (1987) A domain structure common to hemopexin, vitronectin, interstitial collagenase, and a collagenase homolog. Protein Sequences Data Anal. 1, 21-26.

(102) Tantos, A., Kalmar, L., and Tompa, P. (2015) The role of structural disorder in cell cycle regulation, related clinical proteomics, disease development and drug targeting. Expert Rev. Proteomics 12, $221-233$.

(103) Tovo-Rodrigues, L., Recamonde-Mendoza, M., Paixao-Cortes, V. R., Bruxel, E. M., Schuch, J. B., Friedrich, D. C., Rohde, L. A., and Hutz, M. H. (2016) The role of protein intrinsic disorder in major psychiatric disorders. Am. J. Med. Genet., Part B 171, 848-860.

(104) Cortese, M. S., Uversky, V. N., and Dunker, A. K. (2008) Intrinsic disorder in scaffold proteins: Getting more from less. Prog. Biophys. Mol. Biol. 98, 85-106.

(105) Uversky, V. N., Oldfield, C. J., and Dunker, A. K. (2005) Showing your ID: intrinsic disorder as an ID for recognition, regulation and cell signaling. J. Mol. Recognit. 18, 343-384.

(106) Dyson, H. J., and Wright, P. E. (2002) Coupling of folding and binding for unstructured proteins. Curr. Opin. Struct. Biol. 12, 5460 .

(107) Dyson, H. J., and Wright, P. E. (2016) Role of Intrinsic Protein Disorder in the Function and Interactions of the Transcriptional Coactivators CREB-binding Protein (CBP) and p300. J. Biol. Chem. 291, 6714-6722. 
(108) Preissner, K. T., Grulichhenn, J., Ehrlich, H. J., Declerck, P., Justus, C., Collen, D., Pannekoek, H., and Mullerberghaus, G. (1990) Structural requirements for the extracellular interaction of plasminogen activator inhibitor 1 with endothelial cell matrix-associated vitronectin. J. Biol. Chem. 265, 18490-18498.

(109) Rezaie, A. R. (1999) Role of exosites 1 and 2 in thrombin reaction with plasminogen activator inhibitor- 1 in the absence and presence of cofactors. Biochemistry 38, 14592-14599.

(110) Ehrlich, H. J., Gebbink, R. K., Keijer, J., Linders, M., Preissner, K. T., and Pannekoek, H. (1990) Alteration of serpin specificity by a protein cofactor. Vitronectin endows plasminogen activator inhibitor 1 with thrombin inhibitory properties. J. Biol. Chem. 265, 1302913035 .

(111) Andreasen, P. A., Egelund, R., and Petersen, H. H. (2000) The plasminogen activation system in tumor growth, invasion, and metastasis. Cell. Mol. Life Sci. 57, 25-40.

(112) Peng, L., Bhatia, N., Parker, A. C., Zhu, Y. H., and Fay, W. P. (2002) Endogenous vitronectin and plasminogen activator inhibitor-1 promote neointima formation in murine carotid arteries. Arterioscler., Thromb., Vasc. Biol. 22, 934-939.

(113) Podor, T. J., Joshua, P., Butcher, M., Seiffert, D., Loskutoff, D., and Gauldie, J. (1992) Accumulation of type 1 plasminogen activator inhibitor and vitronectin at sites of cellular necrosis and inflammation. Ann. N. Y. Acad. Sci. 667, 173-177.

(114) Tomasini, B. R., Owen, M. C., Fenton, J. W., and Mosher, D. F. (1989) Conformational lability of vitronectin: induction of an antigenic change by alpha-thrombin-serpin complexes and by proteolytically modified thrombin. Biochemistry $28,7617-7623$.

(115) Minor, K. H., and Peterson, C. B. (2002) Plasminogen activator inhibitor type 1 promotes the self-association of vitronectin into complexes exhibiting altered incorporation into the extracellular matrix. J. Biol. Chem. 277, 10337-10345.

(116) Okumura, Y., Kamikubo, Y., Curriden, S. A., Wang, J. Y., Kiwada, T., Futaki, S., Kitagawa, K., and Loskutoff, D. J. (2002) Kinetic analysis of the interaction between vitronectin and the urokinase receptor. J. Biol. Chem. 277, 9395-9404.

(117) Deng, G., Royle, G., Wang, S., Crain, K., and Loskutoff, D. J. (1996) Structural and functional analysis of the plasminogen activator inhibitor-1 binding motif in the somatomedin B domain of vitronectin. J. Biol. Chem. 271, 12716-12723.

(118) Seiffert, D., and Loskutoff, D. J. (1991) Kinetic analysis of the interaction between type 1 plasminogen activator inhibitor and vitronectin and evidence that the bovine inhibitor binds to a thrombin-derived amino-terminal fragment of bovine vitronectin. Biochim. Biophys. Acta, Protein Struct. Mol. Enzymol. 1078, 23-30.

(119) Lawrence, D. A., Palaniappan, S., Stefansson, S., Olson, S. T., FrancisChmura, A. M., Shore, J. D., and Ginsburg, D. (1997) Characterization of the binding of different conformational forms of plasminogen activator inhibitor-1 to vitronectin - Implications for the regulation of pericellular proteolysis. J. Biol. Chem. 272, 7676-7680. (120) Jensen, J. K., Durand, M. K. V., Skeldal, S., Dupont, D. M., Bodker, J. S., Wind, T., and Andreasen, P. A. (2004) Construction of a plasminogen activator inhibitor-1 variant without measurable affinity to vitronectin but otherwise normal. FEBS Lett. 556, 175-179. 